\title{
Unveiling the Fluorescence Origin in Pyrene Derived Graphene Quantum Dots: A Single Particle Level Analysis
}

\author{
Gayatri Batra, ${ }^{1,2}$ II Shubham Sharma, ${ }^{1,2}$ II Kush Kaushik, ${ }^{1,2}$ Chethana Rao, ${ }^{1,2}$ Pawan Kumar, \\ ${ }^{3,4}$ Krishan Kumar, ${ }^{1,2}$ Subrata Ghosh, ${ }^{1,2}$ Deep Jariwala, ${ }^{3}$ Eric A. Stach, ${ }^{4}$ Aditya Yadav ${ }^{1,2} *$ \\ and Chayan Kanti Nandi, ${ }^{1,2}$
}

${ }^{1}$ School of Basic Sciences, Indian Institute of Technology, Mandi, HP-175001, India.

${ }^{2}$ Advanced Materials Research Centre, Indian Institute of Technology, Mandi, HP-175001, India.

${ }^{3}$ Department of Electrical and Systems Engineering, University of Pennsylvania, Philadelphia, PA-19104

${ }^{4}$ Department of Materials Science and Engineering, University of Pennsylvania, Philadelphia, PA-19104

II Gayatri Batra and Shubham Sharma have contributed equally to the work

\begin{abstract}
The bottom-up approach has been the preferred route for large-scale synthesis of graphene quantum dots (GQDs). However, the structure and origin of photoluminescence in these dots synthesized by the bottom-up approach are still a subject of debate. Here, using a series of separation techniques like solvent extraction, column chromatography, gel electrophoresis and dialysis, we present three distinct fluorescent materials in GQDs synthesized from pyrene, a well-known precursor molecule. The subtle balance between the extent of graphitization and molecular fluorophores determines the nature of fluorescence emission in GQDs verified using a suite of optical and vibrational spectroscopy techniques in combination with electron microscopy. The single-particle level emission properties strongly support our observation. Using cell imaging studies, we also verify that all three materials are suitable for fluorescent staining of biological samples. Our results resolve a long-standing debate on the true structural character of GQDs and their source of emission.
\end{abstract}

Keywords: Graphene quantum dots, bottom-up synthesis, pyrene derivative, molecular fluorophore, single particle spectroscopy. 


\section{Introduction}

Graphene Quantum Dots (GQDs) have gained tremendous interest for applications in bioimaging, optoelectronics, photovoltaics etc ${ }^{1-7}$ owing to their chemical stability and low toxicity. By definition, a GQD consists of an atomically thin crystalline $\mathrm{sp}^{2}$ hybridized single graphene layer with armchair and/or zigzag edges.$^{8-10}$ However, even nanocrystals, particles and dots of amorphous and multilayered graphitic carbon (up to 7-8 layers) are often referred to as GQDs in literature. ${ }^{11-16}$ In many of the literature reported, examples of GQDs contain oxygen-rich defects and are also referred to as graphene oxide quantum dots (GOQDs) or partially reduced rGOQDs. ${ }^{13}$ Various functional groups ranging from epoxy, hydroxy, oxide and even carboxyl functional groups embedded on the $\mathrm{sp}^{2}$ graphitic core have been reported. ${ }^{17-20}$ Top-down and bottom-up approaches are the two main approaches for the synthesis of GQDs. ${ }^{21}$ In the top-down method, bulk graphitic materials are used as precursors which are cut into small-sized GQDs. ${ }^{11,22}$ Unfortunately, this method often produces material with inhomogeneous size distributions. One of the most intriguing properties of GQDs is their excitation dependent multicolor photoluminescence. ${ }^{23,24}$ Several mechanisms have been proposed to explain these unusual emission characteristics. While the quantum confinement induced bandgap opening and edge state induced emission have been proposed as plausible explanations, the widely varying emission mechanisms of the GQDs is still a subject of investigation and debate.

Recently, bottom-up approaches have been extensively studied to synthesize high quantum yield (QY) GQDs and carbon nanodots (CNDs), another class of carbon-based fluorescent materials. ${ }^{25,26}$ These are synthesized from small organic molecules via solvothermal and microwave synthesis. In these approaches, the reaction conditions such as temperature, reaction time, and precursor molecule play a significant role in determining the actual amount of GQDs or CNDs in the synthesis products. ${ }^{27,28}$ For example, upon increasing the temperature or reaction time, the amount of graphitization was found to be increased and a clear shift from excitation independent to excitation dependent photoluminescence with a decrease in QY was observed. On the other hand, while the actual problem on the origin of excitation wavelength dependent multicolor photoluminescence is still elusive, several recent reports have shown excitation independent photoluminescence in these dots ${ }^{22,29-32}$. Several hypotheses including the presence of molecular fluorophores and/or their aggregated structure, quasi GQDs (molecular fluorophores attached to the graphitic core) or polymer 
dots, which are produced as by-products or even as the sole product have been proposed to be present in the synthesis products that contribute to the luminescence. ${ }^{33-35}$ Therefore, elucidating the structure-luminescence property relation in GQDs, an outlying and critical question in the field that needs to be thoroughly addressed.

Here, by using a series of separation techniques like solvent extraction, gel-electrophoresis, thin-layer and column chromatography, we reveal that the synthesis products of GQDs as derived from pyrene (a commonly used precursor molecule in the bottom-up synthetic approach) have three distinct emissive components in the synthesis mixture with distinct chemical structure and optical properties. These components showed bright green, greenish brown and deep brown color, when illuminated by $365 \mathrm{~nm}$ UV light. They have different emissions, QY and have different weight percentages in the synthesis mixture. The bright green color material is supposed to be monomer and their isomers of hydroxyl $(\mathrm{OH}) /$ carboxylic acid $(\mathrm{COOH})$ functionalized pyrene derivatives with the highest QY. The greenish brown material is supposed to be quasi GQDs like structure with moderate QY and the deep brown color material is a pure GQDs like structure with the lowest QY. Singleparticle level analysis shows that emission characteristics such as photon counts/cycle, ONOFF switching cycle, and single-particle blinking were all different in these components and thus strongly supporting the distinct nature of the materials.

\section{Results and discussion}

\section{Synthesis and characterization of the pyrene derived GQDs:}

GQDs were synthesized via the hydrothermal method by following a reported protocol for the gram-scale synthesis using pyrene as the precursor molecule. ${ }^{36}$ The detailed synthesis procedure of GQDs is discussed in the supporting information. Briefly, pyrene was converted to 1,3,6-trinitropyrene (TNP) using nitration of $1 \mathrm{~g}$ pyrene in $80 \mathrm{ml}$ nitric acid $\left(\mathrm{HNO}_{3}\right)$ at $80{ }^{\circ} \mathrm{C}$ for overnight (Figure 1a). The optical and chemical characterization of both pyrene and TNP are presented in Figure S1-S6. In the next step, $0.15 \mathrm{~g}$ of TNP was added to $60 \mathrm{ml} 0.2 \mathrm{M} \mathrm{NaOH}$ solution and probe sonicated for $2 \mathrm{~h}$ to disperse the TNP in the solution. The mixture was then transferred to a hydrothermal reactor and heated at $200{ }^{\circ} \mathrm{C}$ for $10 \mathrm{~h}$. After cooling down the mixture to room temperature, it was then filtered through a $0.22 \mu \mathrm{m}$ polyvinylidene difluoride microporous membrane to remove large debris and unreacted material, if any. The obtained solution was freeze-dried to get solid brown GQDs.

The as-synthesized GQDs (crude), upon dissolving into water appeared light brown when examined through the naked eye but changed to green color under $365 \mathrm{~nm} \mathrm{UV-light}$ 
illumination (Figure 1a; top right). Typically, in GQDs, those are synthesized via top-down method from solid graphitic precursor material (details in supporting information), the absorption spectra show exponential decay with maximum absorption below $400 \mathrm{~nm}$. The spectrum generally consists of $\pi-\pi *$ core state transition below $300 \mathrm{~nm}$ and $\mathrm{n}-\pi^{*}$ surface/edge states transitions $(\mathrm{C}-\mathrm{O}, \mathrm{C}=\mathrm{O})$ in the range of $300-400 \mathrm{~nm}$. Along with the above-discussed absorption features, the absorption spectra of the pyrene-derived GQDs show intense vibronic progression peaks above $400 \mathrm{~nm}$ (Figure 1b). These peaks have high similarity with molecular state absorption. The appearance of these new peaks suggests that apart from the core and edge state absorptions, the pyrene-derived GQDs have an additional molecular state like absorption. ${ }^{37-39}$

The emission spectra (Figure 1c) showed excitation wavelength independent emission with a maximum peak at $515 \mathrm{~nm}$ and a shoulder peak at $450 \mathrm{~nm}$. This is quite unusual as GQDs are reported to show excitation wavelength dependent emission spectra, which is also a topic of debate. It is interesting to mention here that while the intensity of the $450 \mathrm{~nm}$ peak is observed to be higher than the $515 \mathrm{~nm}$ peak when excited within 300-380 nm, a switchover of intensity profile from $450 \mathrm{~nm}$ to $515 \mathrm{~nm}$ occurs when excited above $400 \mathrm{~nm}$. The observed excitation wavelength independent emission features at $515 \mathrm{~nm}$ have a close resemblance with emission spectra for organic molecular fluorophore, which abode by the fundamental rules of excited state emission. ${ }^{40}$ As a result, in correlation with the absorption spectra and its transitions, the emission spectral features appearing at $450 \mathrm{~nm}$ in our case likely arise from the core and edge state emission, while the $515 \mathrm{~nm}$ emission features are likely molecular state-like emission. The measured fluorescence lifetime of the synthesized mixture was found to be 3.12 ns (Figure S7).

To further understand and isolate the source of emission, we carried out several structural characterizations to get a deeper insight into the atomic structure, chemical bonding and composition of the material. Powder x-ray diffraction (PXRD) of the sample shows a broad peak at 26 degrees, a typical signature for GQDs (Figure 1d). Transmission electron microscope (TEM) data show a closely homogeneous particle size distribution with a diameter of $\sim 4 \mathrm{~nm}$ and with d spacing of $0.21 \mathrm{~nm}$ (Figure 1e). The Fast Fourier transform (FFT) pattern shows bright spots with a hexagonal arrangement. All these data suggest the formation of crystalline GQDs. On the contrary, in Raman spectroscopy, instead of the characteristic D (defect) and G (graphitic) vibrational bands for GQDs, intense fluorescence background was observed (Figure 1f). Surprisingly, the baseline correction of the spectrum resulted in the desired D and $\mathrm{G}$ bands, ${ }^{41}$ although with lower intensity than expected. It is 
worth pointing out here that the intensity of $\mathrm{D}$ band is almost half of the $\mathrm{G}$ band, suggesting a higher degree of crystallinity in the material. Nevertheless, the observed strong fluorescence background suggests that the synthesized GQDs have a substantial amount of highly emissive impurities such as molecular fluorophores, along with the presence of graphitic material in the synthesis mixture. We carried out both Fourier transform infrared (FTIR) and X-ray photoelectron spectroscopy (XPS) measurements to extract detailed information on the surface and buried functional groups in the synthesized GQD products. Figure 1g shows the comparison of the FTIR spectra of the synthesized GQDs with TNP for a better understanding of the evolution of the functional groups. A broad peak at $\sim 3430 \mathrm{~cm}^{-1}$ (absent in TNP) in the FTIR spectrum and $\sim 1262 \mathrm{~cm}^{-1}$ peak corresponds to $\mathrm{OH}$ vibrational band (Figure 1g). The peaks at $1587 \mathrm{~cm}^{-1}$ and $1450 \mathrm{~cm}^{-1}$ could be attributed to the $\mathrm{C}=\mathrm{C}$ bond. The broad peak at $\sim 1650 \mathrm{~cm}^{-1}$, which is missing in TNP, supports the presence of COOH group functional groups in the material. The survey XPS spectrum shows strong signals of C1s at $285.29 \mathrm{eV}$ and $\mathrm{O} 1 \mathrm{~s}$ peaks at $532.05 \mathrm{eV}$ (Figure S8). The high-resolution spectrum of C1s and O1s confirms the $\mathrm{C}=\mathrm{C}, \mathrm{C}=\mathrm{O}, \mathrm{C}-\mathrm{OH}$ and $\mathrm{COOH}$ functional groups, as observed in the FTIR spectra. ${ }^{42-45}$ It is to be noted here that the absence of the peaks for both $\mathrm{NO}_{2}$ symmetric (1348 $\left.\mathrm{cm}^{-1}\right)$ and $\mathrm{NO}_{2}$ asymmetric $\left(1518 \mathrm{~cm}^{-1}\right)$ in the GQDs FTIR spectra, the appearance of a broad peak at $\sim 1650 \mathrm{~cm}^{-1}$, a broad peak ranging from $2700-3700 \mathrm{~cm}^{-1}$ and the absence of $\mathrm{N} 1 \mathrm{~s}$ in XPS spectrum confirms the complete conversion of TNP to GQDs.

\section{Purification and characterization of as-synthesized GQDs:}

The observed molecular state like transitions both in absorption and emission spectra, excitation wavelength independent emission spectra and strong fluorescence background in Raman spectrum encouraged us to carry out a systematic purification of the synthesized GQDs and characterize each of the separated components to pinpoint the structure-emission property relations. We performed various purification techniques such as solvent extraction, gel-electrophoresis as well as thin-layer and column chromatography. In the solvent extraction method, the crude product was dissolved in $\mathrm{MeOH}$. The dissolved products were taken away every 24 hours by adding a fresh solvent set for eight days. It was found that a small amount $(\sim 3 \%)$ of the products remain entirely undissolved (Figure 2a). We then carried out thin-layer chromatography (TLC) of the $\mathrm{MeOH}$ dissolved material using a 4:1 ratio of $\mathrm{DCM} / \mathrm{MeOH}$ as mobile phase. Two separate spots were clearly visible when TLC paper was illuminated under $365 \mathrm{~nm}$ UV light (Figure 2a; right). Surprisingly, the TLC of the $\mathrm{MeOH}$ insoluble product left with only one immovable component. Next, we carried out 
column chromatography of the $\mathrm{MeOH}$ soluble products using silica as a stationary and $\mathrm{DCM} / \mathrm{MeOH}$ as a mobile phase. Here, two separate components were eluted out from the column at 10:1 (32\% weight of total crude products) and 5:1 (65\% weight of total crude products) ratios of $\mathrm{DCM} / \mathrm{MeOH}$, respectively (Figure 2b). Unfortunately, we could not perform column chromatography of the $\mathrm{MeOH}$ insoluble material as it clogged the column. Nevertheless, it can be concluded that the as-synthesized GQDs product contains three different components with their different polarities and most probably may present as different optical and chemical entities. Inset of Figure $\mathbf{2 c}$ and $2 \mathbf{e}$ represents the normal and $365 \mathrm{~nm}$ UV light illuminated color of the components. The observed $365 \mathrm{~nm}$ UV illuminated colors of the components are depicted as bright green, greenish brown and deep brown.

\section{Optical characterization of the purified components:}

We carried out detailed optical and chemical characterization for each of these components. The optical measurements were done both in well-dispersed bulk solutions and at singleparticle level (detail of experimental procedures are in the supporting information and the results are discussed later). The absorption spectra of all three components are presented in Figure 2c. Remarkably distinct absorption spectral features for all of the three components were observed. The absorption spectra, similar to Figure 1b, categorically could be assigned to three different regions. The greenish brown component has intense absorption bands both below $300 \mathrm{~nm}(\sim 262 \mathrm{~nm})$ and above $400 \mathrm{~nm}$, respectively. The absorption in the range of $300-400 \mathrm{~nm}$ is not prominent for this component. The zoomed version (Figure 2d) of the absorption spectra shows that the bright green component resembles the absorption spectra closely with the greenish brown component with a little variation in the vibronic bands intensity above $400 \mathrm{~nm}$. On the other hand, the deep brown component has strong absorption below $400 \mathrm{~nm}$, as typically observed in GQDs, with a hinged peak at $236 \mathrm{~nm}$ and $355 \mathrm{~nm}$. In addition, it has a much less structured absorption after $400 \mathrm{~nm}$, although with a small hinged peak at around $485 \mathrm{~nm}$. All of the above observations suggested that all these components are fundamentally different by their ground state structure.

Next, the emission spectra of all three components were recorded. The normalized emission spectra (Figure 2e) show a prominent and gradual redshift of the emission maxima from bright green to greenish brown to deep brown color components of the GQDs products when excited at a fixed wavelength of $460 \mathrm{~nm}$. While the emission maxima obtained at $515 \mathrm{~nm}$ for the bright green component, the emission maxima obtained for greenish brown and deep brown components are at $525 \mathrm{~nm}$ and $541 \mathrm{~nm}$ respectively. We calculated the absolute QY by 
the integrated sphere method (supporting information). A substantial decrease in the QY from bright green $(\mathrm{QY}=12 \%)$ to greenish brown $(\mathrm{QY}=8 \%)$ to deep brown component $(\mathrm{QY}=$ $0.8 \%$ ) was observed. The bright green and greenish brown components showed an average lifetime of 4.3 and $3.8 \mathrm{~ns}$, while the average lifetime for the deep brown component was 0.41 ns (Figure 2f). The decrement in the lifetime from bright green (4.3 ns) to the greenish brown and deep brown components (3.8 ns and $0.41 \mathrm{~ns}$ ) and the decrement in the QY suggested decreasing radiative decay rate and increasing non-radiative decay rate.

\section{Raman and TGA measurement of the purified components:}

The emission spectral shifting in Figure 2e, at first glance, could be proposed as the sizedependent quantum confinement effect reported earlier in GQDs. However, the measured Raman spectra and the thermogravimetric analysis (TGA) showed interesting and contrasting results. One of the most intriguing observations in Raman spectra (Figure 2g) is that both the greenish brown and deep brown components show prominent D and G bands, while the bright green component only shows a fluorescence background. Unlike the crude sample, the bright green component shows no signatures of the $\mathrm{D}$ and $\mathrm{G}$ bands even after baseline correction. On the other hand, the bright green component shows (Figure 2h) a gradual but significant mass loss (more than 60\%) within $500{ }^{\circ} \mathrm{C}$ and then a prolonged mass loss till 1000 ${ }^{\circ} \mathrm{C}$ in TGA measurement. It is to be pointed out here that the precursor molecule pyrene showed a complete mass loss at $200{ }^{\circ} \mathrm{C}$ and TNP showed extensive mass loss at around 350 ${ }^{\circ} \mathrm{C}$ (Figure S9). Based on observed results, the bright green component is attributed to a pyrene derived molecular fluorophore. Conversely, the deep brown component displays a very high thermal stability. The greenish brown component showed lesser stability than deep brown but more stable than the bright green component.

\section{Structural analysis by TEM, STEM and SEM:}

For an in-depth structural characterization, we carried out transmission electron microscopy (TEM) and aberration-corrected (probe) scanning TEM (STEM) of all three components for direct visualization of the structure of our products down to the atomic resolution. TEM images of the bright green component doesn't show any particle features specific or analogous to GQDs. Instead, it depicts a variety of aggregated structures. Images of distinct morphologies were observed for the bright green component under different environmental conditions. For example, it showed either stacked thread or ribbon-like structures under a TEM (Fig. 3a, b). Such structures are likely aggregations of the molecular fluorophore. 
Various aggregated morphologies made up of the bright green component are further confirmed by scanning electron microscope (SEM) images. The SEM images presented in Figure 3g-i clearly show long thread, ribbon, petal, or flower-like structures. The STEM image (Figure 4a-c), on the other hand, showed a highly bright white shadow throughout the lacey carbon grids without forming any dot-like structure. Bright shadow in the dark field STEM images suggests the presence of non-crystalline material. In addition, the material gets either burned or appeared blurry in contrast after few continuous scans during STEM imaging, supporting a less stable organic fluorophore structure. The greenish brown component showed fewer dot-like structures in some places of the grid under TEM (Figure 3c, d). The STEM images showed the highly bright white shadow throughout the grid with few dots like structures (Figure 4d-f). These observations suggest the formation of GQDs with an abundant presence of fluorophore attached to it. In fact, on free-hanging places in the lacey grid, the greenish brown component is attached to these organic fluorophores, which do not allow imaging at atomic-scale resolution (Figure S10). On the contrary, the deep brown component showed a huge amount of GQDs with an average size of $3.5 \mathrm{~nm}$ under TEM (Figure 3e, f). The high-resolution images showed distinct inter-layer spacing of $0.21 \mathrm{~nm}$. The STEM images showed the explicit crystalline nature of the GQDs with the size of $3.5 \mathrm{~nm}$ (Figure 4g-h). The cross-sectional view in one of the high-angle annular dark-field imaging (HAADF) STEM image (Figure 4i) shows direct observation of the synthesized GQDs per their reported atomic structure in the appearing stacked form.

\section{Excitation wavelength dependent emission and structural analysis:}

A defining feature of GQDs is their excitation wavelength dependent emission spectra. We measured the excitation wavelength dependent fluorescence from each of the three separated components (Figure 5). Surprisingly, instead of the commonly observed excitation dependent emission, all three components showed excitation wavelength independent emission with a peak maximum above $500 \mathrm{~nm}$. The bright green component showed intense emission above $500 \mathrm{~nm}$ with a negligible emission below this wavelength upon excitation from 280 to 500 $\mathrm{nm}$ (Figure 5a). The greenish brown component also showed intense emission above 500 $\mathrm{nm}$. However, a moderately intense but structured emission was also observed below $500 \mathrm{~nm}$ (Figure 5b). On the other hand, the deep brown component showed almost equally intense emission both above and below $500 \mathrm{~nm}$ (Figure 5c). It is worth mentioning that, irrespective of the excitation wavelength, the bright green component always showed the emission peak at $515 \mathrm{~nm}$, increasing its intensity from 320 to $480 \mathrm{~nm}$ excitation. On the contrary, the greenish 
brown and deep brown components showed prominent emission intensity below $500 \mathrm{~nm}$ when excited within 320 to $400 \mathrm{~nm}$ and a switchover of the intensity happened (from below $500 \mathrm{~nm}$ to above $500 \mathrm{~nm}$ emission) at around $400 \mathrm{~nm}$ or above excitation. From the abovementioned spectral features, it could be concluded that, in all the components, the excitation independent emission mainly arises due to molecular fluorophore state emission, while the edge and core state is increased in greenish brown component and maximum in deep brown component. We were not able to perform chemical characterizations, most probably due to the presence of a large number of isomers of pyrene derivatives. Although the measured XPS spectra (Figure S11 and S12) of both the bright green and greenish brown components strongly support the presence of a large amount of $\mathrm{OH}$ and $\mathrm{COOH}$ functional groups in the sample. In addition, the time-resolved fluorescence anisotropy measurement provided the hydrodynamic diameter of the bright green component as $0.8 \mathrm{~nm}$, which is actually the size of a molecular fluorophore (Figure S13). From all the above data, it could be concluded that the bright green component is $\mathrm{OH} / \mathrm{COOH}$ functionalized pyrene derivative molecular fluorophore. The greenish brown component is assigned as a quasi-GQDs like structure, where the molecular fluorophores are attached to the edges of the core of GQDs (Figure 5e). A similar theoretical interpretation of the monomeric and dimeric forms of perylene on the surface of CNDs has been reported recently. ${ }^{46}$ It is to be pointed out here that, this component has the maximum weight percentage (65\%) with a high QY of $8 \%$ and a high lifetime of 3.8 ns. On the other hand, the deep brown component is the real crystalline GQDs with very low QY and lesser fluorophores are attached to it (Figure 5f). In addition, it has little excitation dependency in the core and edge states emission (Figure 5c). Based on the above observations, it could be concluded that a large amount of highly intense bright green molecular fluorophore (weight percentage of 32\%) with QY of $12 \%$ mainly dictates the green color emission in pyrene derived GQDs as presented in Figure 1.

We also carried out the gel electrophoresis separation and dialytic separation of the synthesized GQDs to check the feasibility of our results. Interestingly, we got exactly the same three emissive components in the agarose gel. The optical absorption and emission spectra closely resemble the column purified bright green, greenish brown, and deep brown color components (Figure S14). The bright green component doesn't show any D and G bands in the Raman spectrum, while they were observed in the greenish brown and deep brown components with intensity increase in the deep brown component. The dialytic separation of the crude GQDs was carried out using $1 \mathrm{kDa}$ membrane (Figure S15). While the dialysate showed all the features of the bright green component, the retentate showed the 
characteristic of greenish brown and deep brown GQDs. It is obvious as $1 \mathrm{kDa}$ membrane will allow coming out only the smaller size molecular fluorophore, keeping the larger size GQDs inside. The measured Raman spectra showed the prominent D \& G bands for retentate but not in the dialysate. The broad peak in PXRD also confirmed the GQDs like structure in the retentate. On the other hand, excitation independent emission was observed for dialysate, while both excitation independent and dependent emission was observed for the retentate.

\section{Single Particle Study of the purified components and cell imaging:}

The single-particle level study of all three separated components were carried out in a homebuilt setup, equipped with 100x, oil immersion, 1.49NA, TIRF objective (Nikon) and $488 \mathrm{~nm}$ laser (Toptica). Details have been discussed in the supporting information. The data were analyzed by Andor Solis software with the help of home-written MATLAB code and Origin 2018 Pro. The measured real-time traces, photon/cycle, ON-OFF switching cycles and single-particle blinking were analyzed and presented in Figure 6. We analyzed approximately hundred real-time trace data for each of these components. Interestingly, photon/cycle values were obtained as 2484 for the bright green component, 746 for the greenish brown component and 536 for the deep brown component (Figure 6a-c). On the other hand, the ON-OFF switching cycles were found to be maximum in greenish brown component (43 cycles), while it was least (18 cycles) for the bright green component and 30 cycles for the deep brown component (Figure 6d-f). The photon/cycle data obtained at the single-particle level is in accordance with the bulk absolute QY, wherein the bright green component has the maximum QY, but the deep brown component has the least QY. Considering the very high switching cycles along with moderate photons/cycle, the greenish brown component has a QY of $8 \%$. We also calculated the single-step bleaching and singleparticle blinking events of these components (Figure 6g-i). Interestingly, blinking events, as high as $\sim 80 \%$, was observed for the greenish brown and deep brown components. On the other hand, the bright green component showed a lesser extent of blinking events (60\%). The high blinking events, high ON-OFF switching suggested that each of these components have the potential application in localization-based super-resolution microscopy. Finally, we checked the bioimaging of these components. The HeLa cells were incubated for 12 hours by each of the three samples. Confocal microscopy images show that all the materials have the high capability to stain and image the cells with high brightness (Figure S16). While the bright green component showed the confocal image only in the green channel, the deep brown GQDs have the capability to get the image in red channel as well. 


\section{Conclusion}

We unveiled three distinct bright green, greenish brown and deep brown colored materials in the pyrene derived bottom-up approach hydrothermally large scale synthesized GQDs. A series of separation techniques were applied to obtain the highly pure materials. We showed that the subtle balance between the extent of graphitization and the amount of the synthesized molecular fluorophores decides the nature of the fluorescence emission. Each separated component has a different emission, QY and a different weight percentage in the synthesis mixture. The bright green color material is supposed to originate from various isomers of $\mathrm{OH} / \mathrm{COOH}$ functionalized pyrene derivatives with the highest QY among all the components and is mainly responsible for the emission color of the crude GQDs products. The greenish brown material is quasi-GQDs like structure with moderate QY and the deep brown color material is pure GQDs like structure with the lowest QY. Single-particle level fluorescence analysis shows that emission characteristics such as photon counts/cycle, ON-OFF switching cycle, and single-particle blinking were all different in these components and thus strongly supporting the distinct nature of the materials. Our results resolve a long-standing debate on the true structural character of GQDs and their source of emission.

\section{Supplementary Materials}

The Supporting Information contains details of the experimental procedures, methods and characterizations.

\section{Acknowledgments}

$\mathrm{CKN}$ is thankful to (SERB) core research grant (CRG) India for the project number CRG/2020/000268 and the facilities of the AMRC and BioX center of IIT Mandi, India. Aditya Yadav thanks the Council of Scientific and Industrial Research, India [CSIR SRF:09/1058(0014)/2019-EMR-I]. Shubham Sharma thanks the University Grants Commission, India [(16-9(June 2019)/2019(NET/CSIR)]. The STEM work was carried out at the Singh Center for Nanotechnology at the University of Pennsylvania which is supported by the National Science Foundation (NSF) National Nanotechnology Coordinated Infrastructure Program grant NNCI-1542153 as well as University of Pennsylvania Materials Research Science and Engineering Center (MRSEC). D.J., E.A.S., and P.K. acknowledge primary support for this work from U. Penn MRSEC seed grant supported by the NSF (DMR-1720530) and NSF DMR Electronic Photonic and Magnetic Materials (EPM) core program grant (DMR-1905853). 


\section{Competing interests}

The authors declare no competing financial interests.

\section{References}

(1) Zhu, S.; Zhang, J.; Qiao, C.; Tang, S.; Li, Y.; Yuan, W.; Li, B.; Tian, L.; Liu, F.; Hu, R.; et al. Strongly Green-Photoluminescent Graphene Quantum Dots for Bioimaging Applications. Chem. Commun. 2011, 47 (24), 6858-6860.

(2) Zheng, X. T.; Ananthanarayanan, A.; Luo, K. Q.; Chen, P. Glowing Graphene Quantum Dots and Carbon Dots: Properties, Syntheses, and Biological Applications. small 2015, 11 (14), 1620-1636.

(3) Zhang, Z.; Zhang, J.; Chen, N.; Qu, L. Graphene Quantum Dots: An Emerging Material for Energy-Related Applications and Beyond. Energy $\backslash \&$ Environ. Sci. 2012, 5 (10), 8869-8890.

(4) Yan, Y.; Gong, J.; Chen, J.; Zeng, Z.; Huang, W.; Pu, K.; Liu, J.; Chen, P. Recent Advances on Graphene Quantum Dots: From Chemistry and Physics to Applications. Adv. Mater. 2019, 31 (21), 1808283.

(5) Kadian, S.; Sethi, S. K.; Manik, G. Recent Advancements in Synthesis and Property Control of Graphene Quantum Dots for Biomedical and Optoelectronic Applications. Mater. Chem. Front. 2021, 5 (2), 627-658.

(6) Liu, Q.; Sun, J.; Gao, K.; Chen, N.; Sun, X.; Ti, D.; Bai, C.; Cui, R.; Qu, L. Graphene Quantum Dots for Energy Storage and Conversion: From Fabrication to Applications. Mater. Chem. Front. 2020, 4 (2), 421-436.

(7) Liu, W.; Li, M.; Jiang, G.; Li, G.; Zhu, J.; Xiao, M.; Zhu, Y.; Gao, R.; Yu, A.; Feng, M.; et al. Graphene Quantum Dots-Based Advanced Electrode Materials: Design, Synthesis and Their Applications in Electrochemical Energy Storage and Electrocatalysis. Adv. Energy Mater. 2020, 10 (29), 2001275.

(8) Zhao, R.; Wang, J.; Yang, M.; Liu, Z.; Liu, Z. Graphene Quantum Dots Embedded in a Hexagonal BN Sheet: Identical Influences of Zigzag/Armchair Edges. Phys. Chem. Chem. Phys. 2013, 15 (3), 803-806.

(9) Hu, W.; Huang, Y.; Qin, X.; Lin, L.; Kan, E.; Li, X.; Yang, C.; Yang, J. RoomTemperature Magnetism and Tunable Energy Gaps in Edge-Passivated Zigzag Graphene Quantum Dots. npj 2D Mater. Appl. 2019, 3 (1), 1-5.

(10) Cheng, S.; Yu, J.; Ma, T.; Peres, N. M. R. Strain-Induced Edge Magnetism at the Zigzag Edge of a Graphene Quantum Dot. Phys. Rev. B 2015, 91 (7), 75410.

(11) Pan, D.; Zhang, J.; Li, Z.; Wu, M. Hydrothermal Route for Cutting Graphene Sheets into Blue-Luminescent Graphene Quantum Dots. Adv. Mater. 2010, 22 (6), 734-738.

(12) Li, Y.; Hu, Y.; Zhao, Y.; Shi, G.; Deng, L.; Hou, Y.; Qu, L. An Electrochemical Avenue to Green-Luminescent Graphene Quantum Dots as Potential ElectronAcceptors for Photovoltaics. Adv. Mater. 2011, 23 (6), 776-780.

(13) Liu, F.; Jang, M.-H.; Ha, H. D.; Kim, J.-H.; Cho, Y.-H.; Seo, T. S. Facile Synthetic Method for Pristine Graphene Quantum Dots and Graphene Oxide Quantum Dots: Origin of Blue and Green Luminescence. Adv. Mater. 2013, 25 (27), 3657-3662.

(14) Liu, R.; Wu, D.; Feng, X.; Müllen, K. Bottom-up Fabrication of Photoluminescent Graphene Quantum Dots with Uniform Morphology. J. Am. Chem. Soc. 2011, 133 (39), 15221-15223.

(15) Lee, J.; Kim, K.; Park, W. I.; Kim, B.-H.; Park, J. H.; Kim, T.-H.; Bong, S.; Kim, C.H.; Chae, G.; Jun, M.; et al. Uniform Graphene Quantum Dots Patterned from SelfAssembled Silica Nanodots. Nano Lett. 2012, 12 (12), 6078-6083. 
(16) Peng, J.; Gao, W.; Gupta, B. K.; Liu, Z.; Romero-Aburto, R.; Ge, L.; Song, L.; Alemany, L. B.; Zhan, X.; Gao, G.; et al. Graphene Quantum Dots Derived from Carbon Fibers. Nano Lett. 2012, 12 (2), 844-849.

(17) Liu, B.; Xie, J.; Ma, H.; Zhang, X.; Pan, Y.; Lv, J.; Ge, H.; Ren, N.; Su, H.; Xie, X.; et al. From Graphite to Graphene Oxide and Graphene Oxide Quantum Dots. Small 2017, 13 (18), 1601001.

(18) Jin, S. H.; Kim, D. H.; Jun, G. H.; Hong, S. H.; Jeon, S. Tuning the Photoluminescence of Graphene Quantum Dots through the Charge Transfer Effect of Functional Groups. ACS Nano 2013, 7 (2), 1239-1245.

(19) Wang, S.; Cole, I. S.; Zhao, D.; Li, Q. The Dual Roles of Functional Groups in the Photoluminescence of Graphene Quantum Dots. Nanoscale 2016, 8 (14), 7449-7458.

(20) Li, Y.; Zhao, Y.; Cheng, H.; Hu, Y.; Shi, G.; Dai, L.; Qu, L. Nitrogen-Doped Graphene Quantum Dots with Oxygen-Rich Functional Groups. J. Am. Chem. Soc. 2012, 134 (1), 15-18.

(21) Bacon, M.; Bradley, S. J.; Nann, T. Graphene Quantum Dots. Part. I\& Part. Syst. Charact. 2014, 31 (4), 415-428.

(22) Zhu, X.; Xiao, X.; Zuo, X.; Liang, Y.; Nan, J. Hydrothermal Preparation of Photoluminescent Graphene Quantum Dots Characterized Excitation-Independent Emission and Its Application as a Bioimaging Reagent. Part. $\backslash \&$ Part. Syst. Charact. 2014, 31 (7), 801-809.

(23) Gan, Z.; Xu, H.; Hao, Y. Mechanism for Excitation-Dependent Photoluminescence from Graphene Quantum Dots and Other Graphene Oxide Derivates: Consensus, Debates and Challenges. Nanoscale 2016, 8 (15), 7794-7807.

(24) Lai, S.; Jin, Y.; Shi, L.; Zhou, R.; Zhou, Y.; An, D. Mechanisms behind Excitation-and Concentration-Dependent Multicolor Photoluminescence in Graphene Quantum Dots. Nanoscale 2020, 12 (2), 591-601.

(25) Wu, X.; Tian, F.; Wang, W.; Chen, J.; Wu, M.; Zhao, J. X. Fabrication of Highly Fluorescent Graphene Quantum Dots Using L-Glutamic Acid for in Vitro/in Vivo Imaging and Sensing. J. Mater. Chem. C 2013, 1 (31), 4676-4684.

(26) Hou, J.; Wang, W.; Zhou, T.; Wang, B.; Li, H.; Ding, L. Synthesis and Formation Mechanistic Investigation of Nitrogen-Doped Carbon Dots with High Quantum Yields and Yellowish-Green Fluorescence. Nanoscale 2016, 8 (21), 11185-11193.

(27) Ehrat, F.; Bhattacharyya, S.; Schneider, J.; Löf, A.; Wyrwich, R.; Rogach, A. L.; Stolarczyk, J. K.; Urban, A. S.; Feldmann, J. Tracking the Source of Carbon Dot Photoluminescence: Aromatic Domains versus Molecular Fluorophores. Nano Lett. 2017, 17 (12), 7710-7716.

(28) Krysmann, M. J.; Kelarakis, A.; Dallas, P.; Giannelis, E. P. Formation Mechanism of Carbogenic Nanoparticles with Dual Photoluminescence Emission. J. Am. Chem. Soc. 2011, 134 (2), 747-750.

(29) Chen, S.; Hai, X.; Xia, C.; Chen, X.-W.; Wang, J.-H. Preparation of ExcitationIndependent Photoluminescent Graphene Quantum Dots with Visible-Light Excitation/Emission for Cell Imaging. Chem. Eur. J. 2013, 19 (47), 15918-15923.

(30) Maiti, S.; Kundu, S.; Roy, C. N.; Das, T. K.; Saha, A. Synthesis of Excitation Independent Highly Luminescent Graphene Quantum Dots through Perchloric Acid Oxidation. Langmuir 2017, 33 (51), 14634-14642.

(31) Ke, C.-C.; Yang, Y.-C.; Tseng, W.-L. Synthesis of Blue-, Green-, Yellow-, and RedEmitting Graphene-Quantum-Dot-Based Nanomaterials with Excitation-Independent Emission. Part. I\& Part. Syst. Charact. 2016, 33 (3), 132-139.

(32) Kuo, W.-S.; Shen, X.-C.; Chang, C.-Y.; Kao, H.-F.; Lin, S.-H.; Wang, J.-Y.; Wu, P.-C. Multiplexed Graphene Quantum Dots with Excitation-Wavelength-Independent 
Photoluminescence, as Two-Photon Probes, and in Ultraviolet--Near Infrared Bioimaging. ACS Nano 2020, 14 (9), 11502-11509.

(33) Soni, N.; Singh, S.; Sharma, S.; Batra, G.; Kaushik, K.; Rao, C.; Verma, N. C.; Mondal, B.; Yadav, A.; Nandi, C. K. Absorption and Emission of Light in Red Emissive Carbon Nanodots. Chem. Sci. 2021, 12, 3615-3626.

(34) Verma, N. C.; Yadav, A.; Nandi, C. K. Paving the Path to the Future of Carbogenic Nanodots. Nat. Commun. 2019, 10 (1), 2391.

(35) Khan, S.; Sharma, A.; Ghoshal, S.; Jain, S.; Hazra, M. K.; Nandi, C. K. Small Molecular Organic Nanocrystals Resemble Carbon Nanodots in Terms of Their Properties. Chem. Sci. 2018, 9 (1), 175-180.

(36) Wang, L.; Wang, Y.; Xu, T.; Liao, H.; Yao, C.; Liu, Y.; Li, Z.; Chen, Z.; Pan, D.; Sun, L.; et al. Gram-Scale Synthesis of Single-Crystalline Graphene Quantum Dots with Superior Optical Properties. Nat. Commun. 2014, 5, 5357.

(37) Shamsipur, M.; Barati, A.; Taherpour, A. A.; Jamshidi, M. Resolving the Multiple Emission Centers in Carbon Dots: From Fluorophore Molecular States to Aromatic Domain States and Carbon-Core States. J. Phys. Chem. Lett. 2018, 9 (15), 4189-4198.

(38) Sharma, A.; Gadly, T.; Neogy, S.; Ghosh, S. K.; Kumbhakar, M. Addition to "Molecular Origin and Self-Assembly of Fluorescent Carbon Nanodots in Polar Solvents." J. Phys. Chem. Lett. 2017, 8 (23), 5861-5864.

(39) Zhu, P.; Tan, K.; Chen, Q.; Xiong, J.; Gao, L. Origins of Efficient Multiemission Luminescence in Carbon Dots. Chem. Mater. 2019, 31 (13), 4732-4742.

(40) Lakowicz, J. R. Principles of Fluorescence Spectroscopy; Springer Science \& Business Media, 2006.

(41) Kumar, G. S.; Roy, R.; Sen, D.; Ghorai, U. K.; Thapa, R.; Mazumder, N.; Saha, S.; Chattopadhyay, K. K. Amino-Functionalized Graphene Quantum Dots: Origin of Tunable Heterogeneous Photoluminescence. Nanoscale 2014, 6 (6), 3384-3391.

(42) Ye, R.; Peng, Z.; Metzger, A.; Lin, J.; Mann, J. A.; Huang, K.; Xiang, C.; Fan, X.; Samuel, E. L. G.; Alemany, L. B.; et al. Bandgap Engineering of Coal-Derived Graphene Quantum Dots. ACS Appl. Mater. I\& interfaces 2015, 7 (12), 7041-7048.

(43) Rajender, G.; Giri, P. K. Formation Mechanism of Graphene Quantum Dots and Their Edge State Conversion Probed by Photoluminescence and Raman Spectroscopy. J. Mater. Chem. C 2016, 4 (46), 10852-10865.

(44) Du, J.; Wang, H.; Wang, L.; Zhu, S.; Song, Y.; Yang, B.; Sun, H. Insight into the Effect of Functional Groups on Visible-Fluorescence Emissions of Graphene Quantum Dots. J. Mater. Chem. C 2016, 4 (11), 2235-2242.

(45) Rabchinskii, M. K.; Dideikin, A. T.; Kirilenko, D. A.; Baidakova, M. V; Shnitov, V. V; Roth, F.; Konyakhin, S. V; Besedina, N. A.; Pavlov, S. I.; Kuricyn, R. A.; et al. Facile Reduction of Graphene Oxide Suspensions and Films Using Glass Wafers. Sci. Rep. 2018, 8 (1), 1-11.

(46) Kundelev, E. V; Tepliakov, N. V; Leonov, M. Y.; Maslov, V. G.; Baranov, A. V; Fedorov, A. V; Rukhlenko, I. D.; Rogach, A. L. Toward Bright Red-Emissive Carbon Dots through Controlling Interaction among Surface Emission Centers. J. Phys. Chem. Lett. 2020, 11 (19), 8121-8127. 


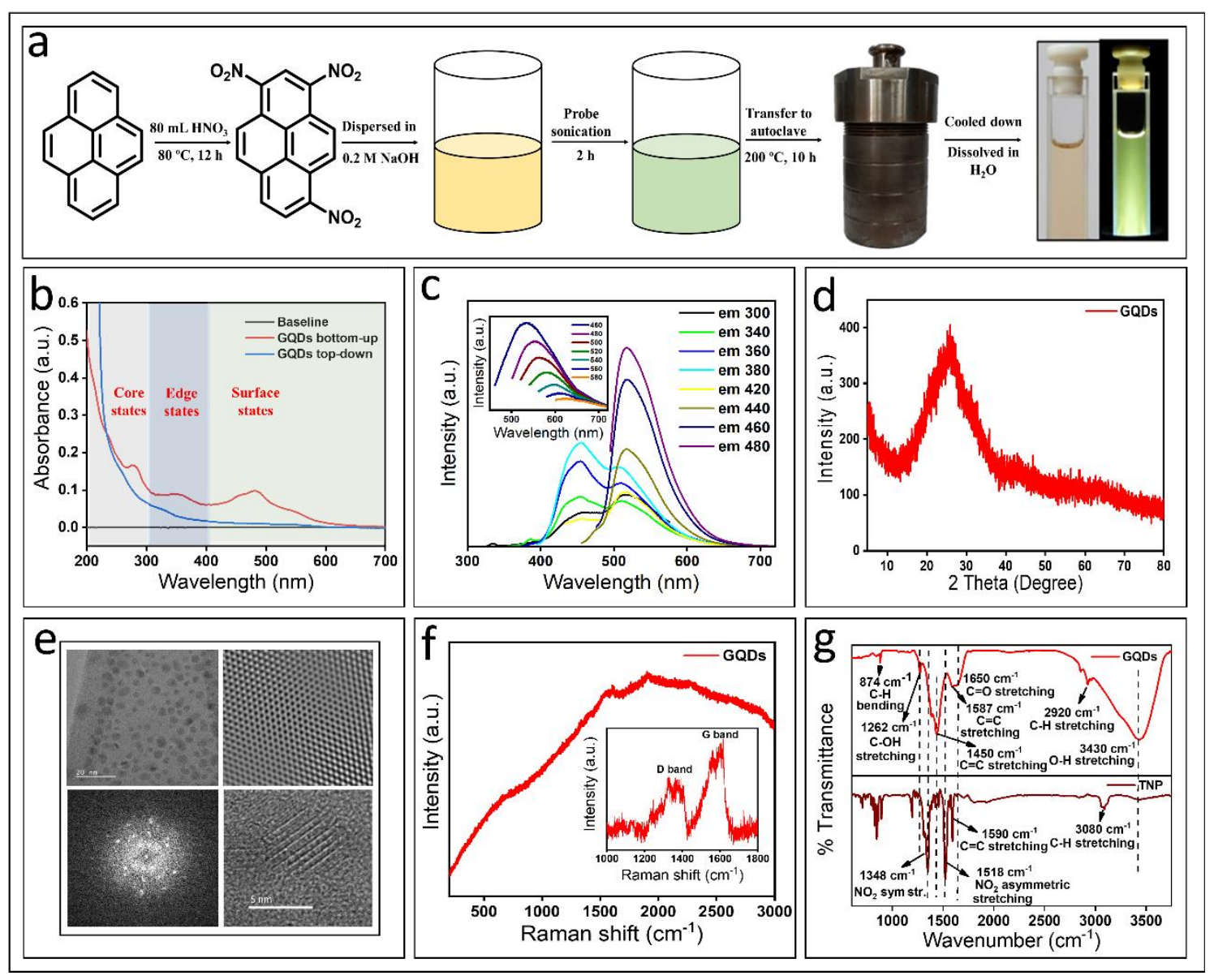

Fig. 1 (a) Schematics of the synthesis of GQDs derived from pyrene. (b) A comparison of the absorption spectra (red color) of GQDs synthesized from pyrene via bottom-up approach and (blue color) from graphite via top-down approach. (c) Excitation independent emission spectra of GQDs derived from pyrene via bottom-up approach, (inset) excitation dependent emission in GQDs synthesis from graphite via top-down approach. (d) PXRD spectrum with a broad peak at $~ 26$ degrees. (e) TEM, inverse FFT, FFT and HRTEM images show the honeycomb crystalline nature of GQDs. (f) Raman spectrum shows the maximum fluorescence background, (inset) shows the signature of typical D and $G$ bands after baseline correction. (g) Comparison of the FTIR spectra of GQDs and TNP. 


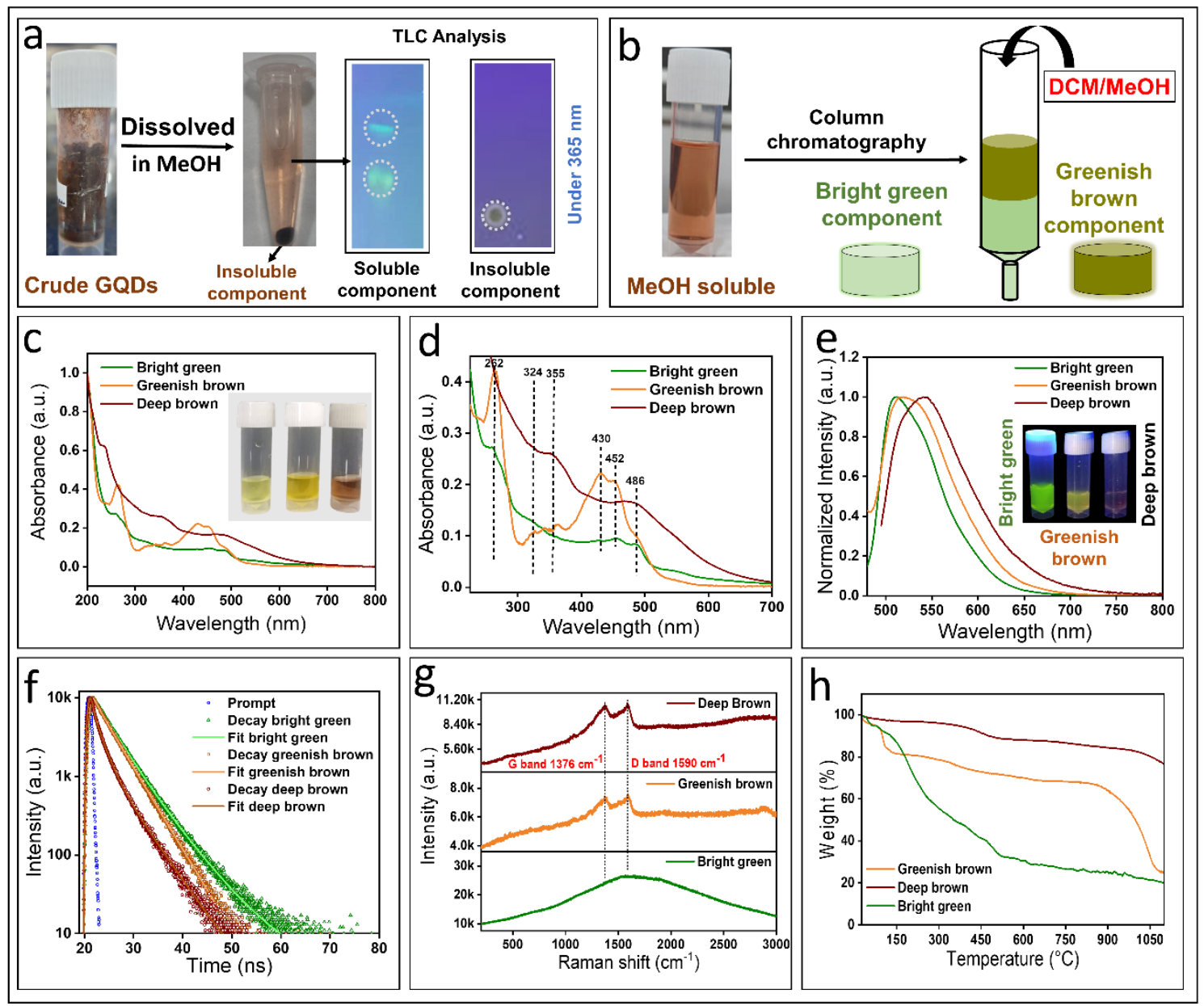

Fig. 2 (a) Detail schematic of the $\mathrm{MeOH}$ solvent extracted separation of crude GQDs. (b) Schematic of column chromatographic separation of $\mathrm{MeOH}$ soluble components. (c) A comparison of the absorption spectra of all three separated components, (inset) naked eye image of the components. (d) Zoomed version of the absorption spectra. (e) Normalized steady-state fluorescence emission spectra of all separated components, (inset) show the 365 UV light illuminated color. (f) Lifetime decay of all three components with bright green component showing maximum lifetime and deep brown component showing the lowest lifetime. (g) Raman spectra of the separated components: bright green component shows only the fluorescence background without $\mathrm{D}$ and $\mathrm{G}$ band while greenish brown and deep brown components show prominent $\mathrm{D}$ and $\mathrm{G}$ bands. (h) TGA spectra suggesting completely different thermal stability of each of the components. 


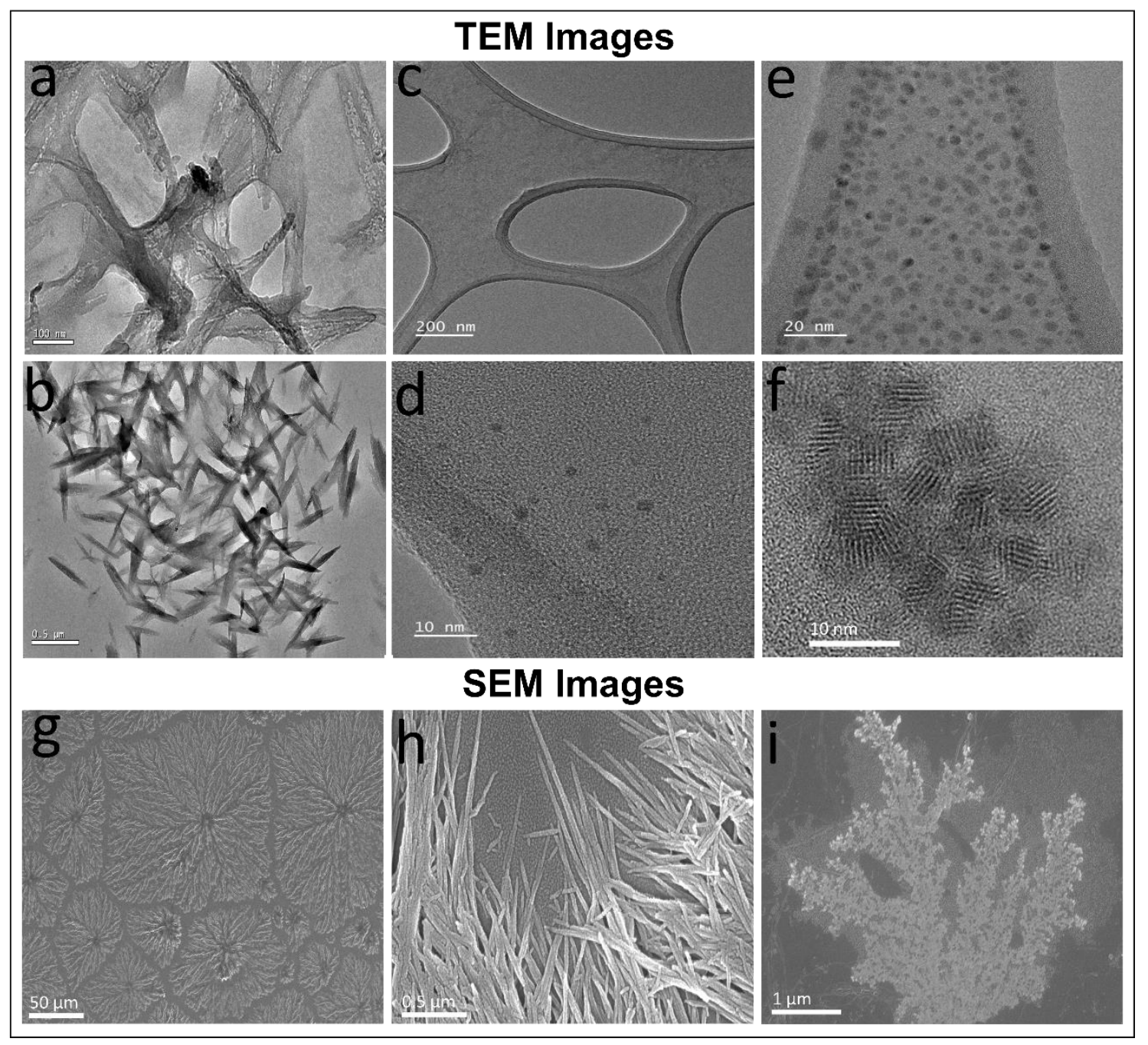

Fig. 3 TEM images of (a-b) bright green, (c-d) greenish brown and (e-f) deep brown component. Bright green component shows different types of aggregated structure under different experimental conditions but without any GQDs formation, the greenish brown component shows little GQDs like structure within the background, deep brown component shows perfect GQDs formation with prominent lattice fringes. (g-i) Different morphological SEM images of the bright green component under different experimental conditions. 


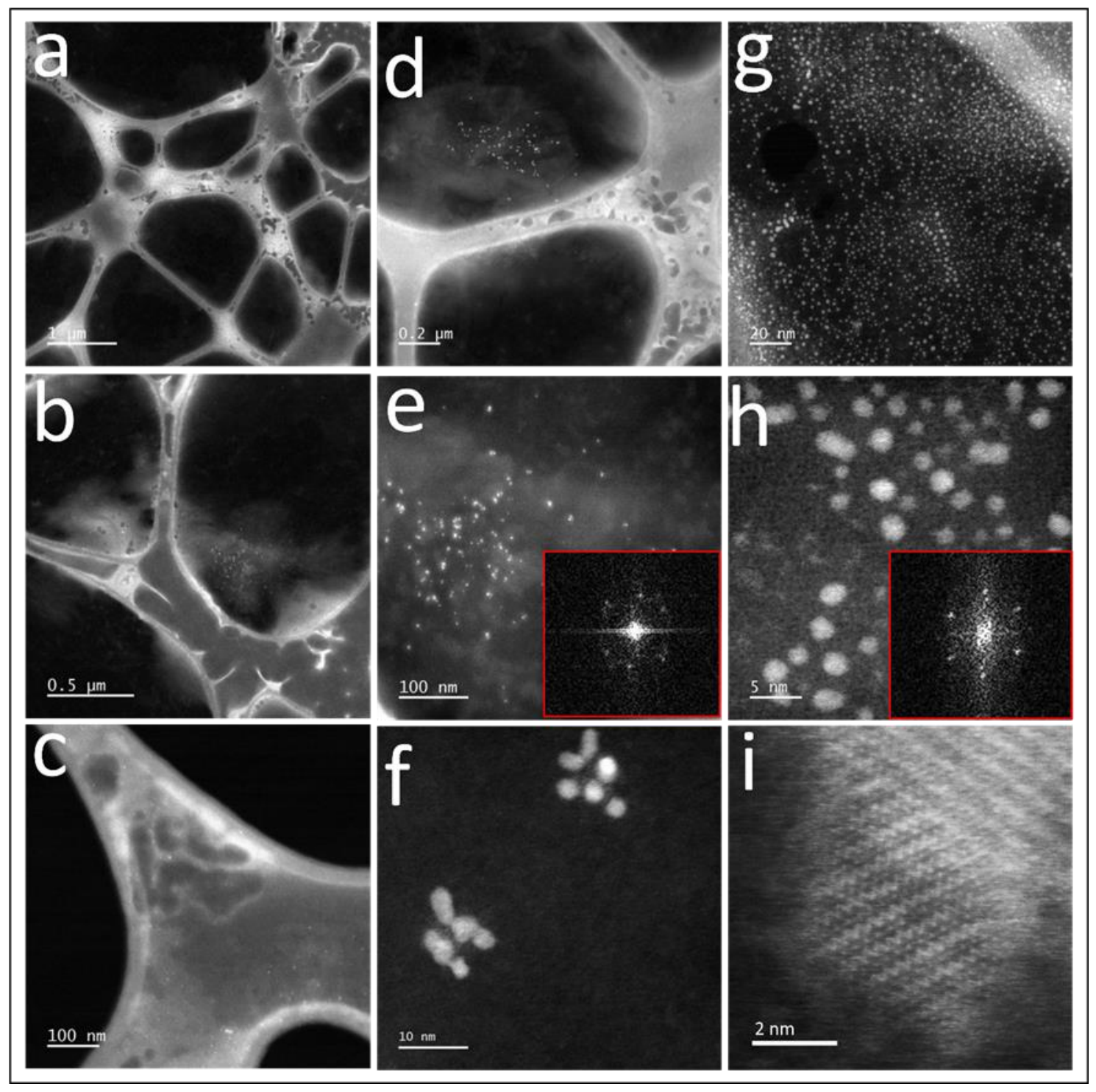

Fig. 4 (a-c) STEM images of the bright green, (d-f) greenish brown and (g-i) deep brown component. (a, d, g) are the low-resolution images and (c, f, i) are the high-resolution images of bright green, greenish brown and deep brown components. The bright green component shows only background without any structured GQDs. The greenish brown component also shows high background with little formation of GQDs like structure, while the deep brown component shows highly ordered GQDs structure. 


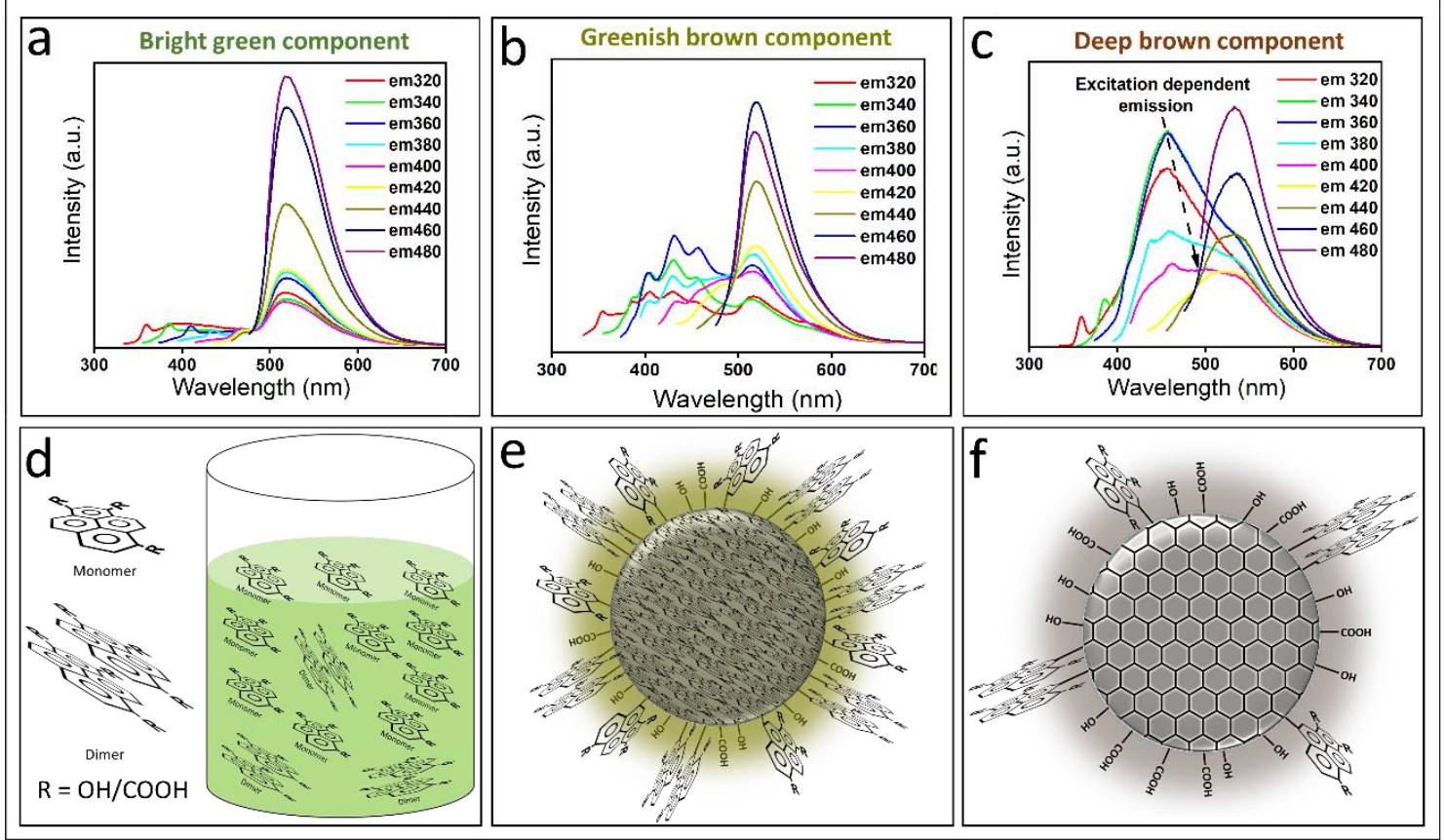

Fig. 5 Excitation wavelength dependent emission spectra: (a) Bright green component shows excitation independent emission only above $500 \mathrm{~nm}$ with a peak maximum at $515 \mathrm{~nm}$. (b) Greenish brown component shows two emission features with excitation independent emission above $500 \mathrm{~nm}$ with a maximum at $525 \mathrm{~nm}$ and structured but excitation independent emission below $500 \mathrm{~nm}$. (c) Deep brown component shows almost equally intense excitation wavelength independent emission with maximum at $541 \mathrm{~nm}$ and excitation dependent emission with a peak maximum at around $450 \mathrm{~nm}$. (d-f) Proposed structure of bright green, greenish brown and deep brown components. 


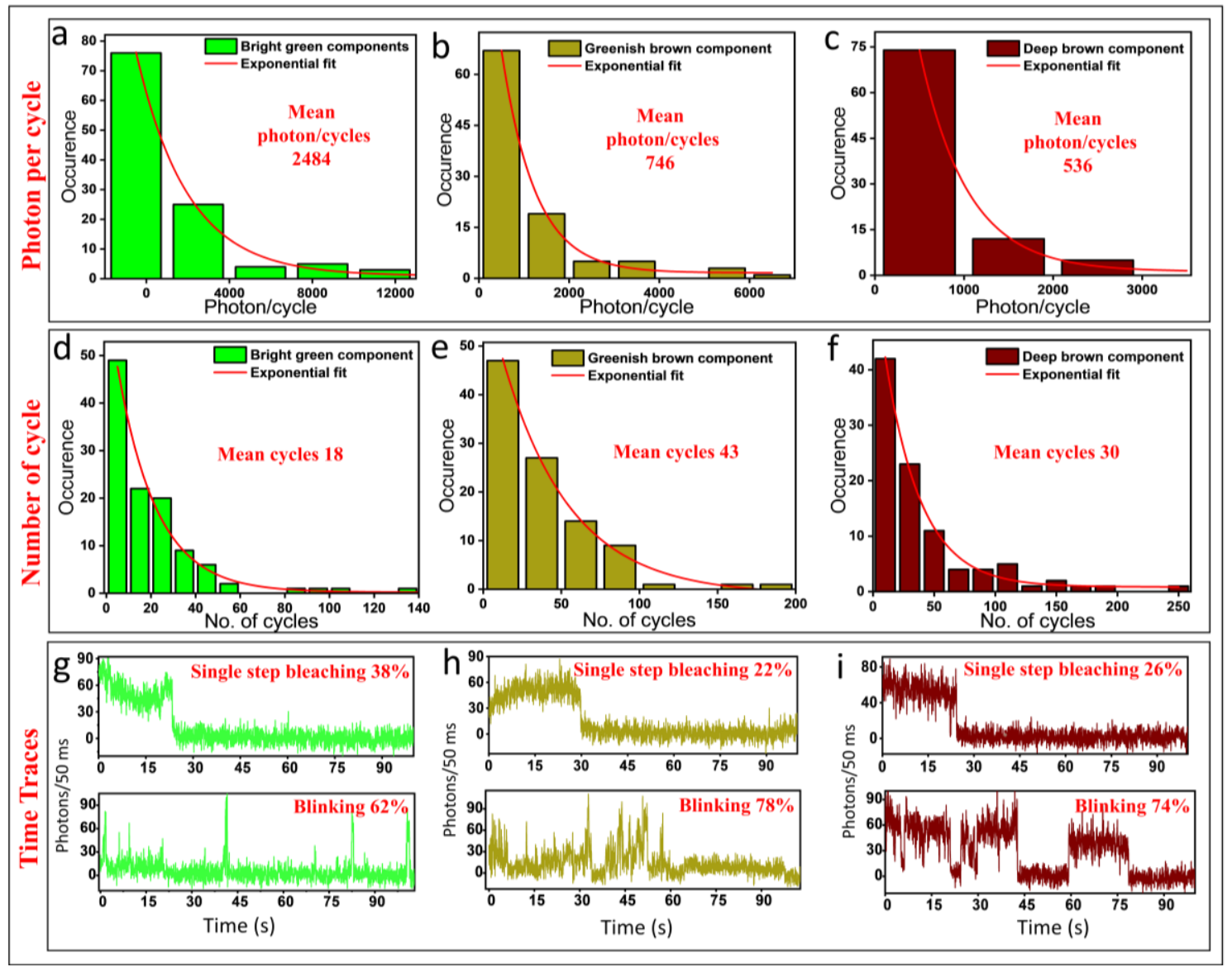

Fig. 6 Measured single particle level (a-c) photons counts/cycle, (d-f) number of ON-OFF switching cycles for each of the separated components. The maximum photon counts/cycle was observed for the bright green component, while least photon counts/cycle was observed for deep brown component. (g-i) Single particle time traces of both single-step bleaching and blinking events. 


\title{
Supporting Information
}

\section{Unveiling the Fluorescence Origin in Pyrene Derived Graphene Quantum Dots: A Single Particle Level Analysis}

\author{
Gayatri Batra, ${ }^{1,2}$ ql Shubham Sharma, ${ }^{1,2}$ II Kush Kaushik, ${ }^{1,2}$ Chethana Rao, 1, 2 Pawan Kumar, \\ ${ }^{3,4}$ Krishan Kumar, ${ }^{1,2}$ Subrata Ghosh, ${ }^{1,2}$ Deep Jariwala, ${ }^{3}$ Eric A. Stach, ${ }^{4}$ Aditya Yadav ${ }^{1,2} *$ \\ and Chayan Kanti Nandi ${ }^{1,2}$ *
}

${ }^{1}$ School of Basic Sciences, Indian Institute of Technology, Mandi, HP-175001, India.

${ }^{2}$ Advanced Materials Research Centre, Indian Institute of Technology, Mandi, HP-175001, India.

${ }^{3}$ Department of Electrical and System Engineering, University of Pennsylvania, Philadelphia, PA-19104

${ }^{4}$ Department of Materials Science and Engineering, University of Pennsylvania, Philadelphia, PA-19104

If Gayatri Batra and Shubham Sharma have equally contributed to the work

\section{Experimental}

\section{Materials:}

All glassware was washed with aqua regia, followed by rinsing several times with doubledistilled water. Pyrene was purchased from Alfa Aesar. $\mathrm{HCl}$ and $\mathrm{HNO}_{3}$ were purchased from fisher scientific. All chemicals were used without further purification. Double-distilled (18.3 $\mathrm{m} \Omega$ ) deionized water (ELGA PURELAB Ultra) was used throughout the entire process.

\section{Characterization}

The UV-Vis absorption spectra were recorded using Shimadzu UV-Vis 2450 spectrophotometer. The spectra were collected using a quartz cuvette having a $10 \mathrm{~mm}$ path length and $1 \mathrm{ml}$ volume. Steady-state fluorescence spectra were recorded on a Horiba spectrophotometer. The fluorescence lifetime measured using Horiba Scientific Delta Flex TCSPC system with pulsed LED Sources. Ludox has been used to calculate IRF for deconvolution of the spectral value. Powder X-ray diffraction (PXRD) pattern was recorded on a Rigaku Smart Lab diffractometer, using $\mathrm{Cu}-\mathrm{K}_{\alpha}$ radiation from $5^{\circ}$ to $80^{\circ}$ with a scanning rate of $2 \%$ min. The particles shape and structure were analyzed by transmission electron 
microscopy (TEM) using FEI Tecnai equipped with a LaB6 source and JEOL (F-200) containing cold-field emission gun, microscopes operating at $200 \mathrm{kV}$. An aberrationcorrected (probe correction) JEOL NEOARM microscope, used for scanning transmission electron microscopy (STEM) operated at an accelerating voltage of $200 \mathrm{kV}$. High-angle annular dark-field (HAADF) STEM images were acquired with $1 \AA$ probe diameter at a convergence angle of 25-29 mrad and the condenser lens aperture was kept at $40 \mu \mathrm{m}$ with a camera length of $4 \mathrm{~cm}$, probe current measured was $120 \mathrm{pAmp}$. The captured STEM images were collected using GATAN GMS (v3) application and associated GATAN dark-field highangle annular detector. The Raman spectrum was measured by the confocal microscope Raman spectrometer (Horiba Scientific, XploRA ONE). Thermal properties were measured by Perkin Elmer Pyris Thermogravimetric (TGA) analyzer under a nitrogen atmosphere with a heating rate of $10{ }^{\circ} \mathrm{C} \min ^{-1}$. A field emission scanning electron microscopy FESEM (NOVA NanoSEM 450) at an accelerating voltage of $10 \mathrm{kV}$ was used to capture SEM images. Fourier transforms infrared (FTIR) spectra were measured using a Perkin-Elmer FTIR spectrophotometer equipped with a horizontal attenuated total reflectance (ATR) accessory containing a zinc selenide crystal and operating at $4 \mathrm{~cm}^{-1}$ resolution. The use of spectral subtraction provided reliably reproducible results. HRMS spectra were recorded on a Bruker impact-HD spectrometer and ${ }^{1} \mathrm{H}$ NMR spectra were recorded on a Jeol-ECX-500 $\mathrm{MHz}$ spectrometer using tetramethylsilane (TMS) as an internal standard. X-ray photoelectron spectroscopy (XPS) measurements were carried out on a NEXSA surface analysis model by Thermo Fisher Scientific using Al-K $\mathrm{K}_{\alpha}(1486.6 \mathrm{eV}) \mathrm{X}$-ray radiation. The XPS data were acquired with a spot size $400 \mu \mathrm{m}$ having a standard lens mode.

\section{Synthesis procedures for GQDs using top-down approach}

\section{Synthesis of Graphene Oxide from Graphite (Hummer's Method):}

Graphite $(2.0 \mathrm{~g})$ was dissolved in $27 \mathrm{~mL}$ of $\mathrm{H}_{2} \mathrm{SO}_{4}$ and sodium nitrate $(0.5 \mathrm{~g})$. The mixture was then stirred at room temperature for $1 \mathrm{~h}$ and $\mathrm{KMNO}_{4}(3 \mathrm{~g})$ was added using ice bath and the mixture was stirred at $40{ }^{\circ} \mathrm{C}$ for $12 \mathrm{~h}$ and Distilled water $(500 \mathrm{~mL})$ was added and stirring was done for $1.5 \mathrm{~h}$ and hydrogen peroxide $(5 \mathrm{~mL})$ was added and the reaction mixture was left undisturbed, and decantation was done, and the as prepared Graphene oxide was washed with $\mathrm{DI}$ and $\mathrm{HCl}$ and dried.

\section{Synthesis of GQDs from GO using NaOH:}

Graphene Oxide $(0.2 \mathrm{~g})$ was dissolved in $5 \mathrm{M} \mathrm{NaOH}(20 \mathrm{~mL})$ and the mixture was then sonicated for 5 minutes and then transferred to a hydrothermal autoclave reactor $(50 \mathrm{~mL})$ and 
heated at $180^{\circ} \mathrm{C}$ for $12 \mathrm{~h}$. The reactor was cooled to room temperature and the reaction mixture was filtered with a $0.22 \mu \mathrm{m}$ polyvinylidene difluoride microporous membrane to remove unreacted part of GO and Dialysis was done to remove salt ions.

\section{Absolute quantum yield measurements using integration sphere method}

The quantum yield $\left(\Phi_{\mathrm{F}}\right)$ of fluorescence is defined as the ratio of the number of emitted photons and the number of absorbed photons during the excitation of a sample under investigation.

$$
\text { Quantum Yield }=\frac{\text { Total emitted } \text { photon }}{\text { Total absorbed photon }}
$$

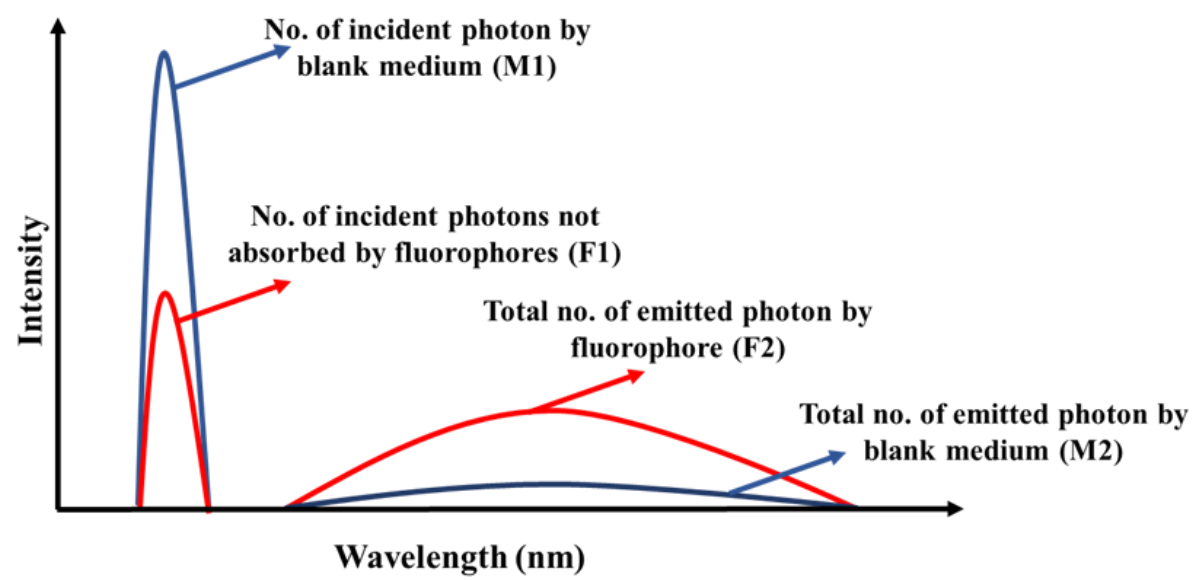

These are the spectra we can record using flourolog during absolute quantum yield.

$$
\text { Absolute Quantum Yield }=\frac{\text { Total emitted photon }(F 2-M 2)}{\text { Total absorbed photon }(M 1-F 1)}
$$

Total emitted photon $=$ Area under F2 region - Area under M2 region

Total absorbed photon $=$ Area under M1 region - Area under F1 region

\section{Single-molecule time trace and photon counts}

All the separated components were spin-coated on a cleaned glass coverslip to collect singlemolecule time traces. The diffraction-limited spots produced due to single-molecule blinking were observed using the 100x Nikon TIRF objective. A $488 \mathrm{~nm}$ diode laser with $40 \mathrm{~mW}$ maximum power was used. An oil immersion Nikon TIRF objective (100x magnification and 1.49 NA) was mounted on a custom build inverted optical microscope (Nikon Ti 
epifluorescence microscope). A $488 \mathrm{~nm}$ high pass Dichroic (AHF Analysentechnik) was used to separate the excitation and emission light, and a bandpass filter of 575/50 is used to further reject the excitation laser and allow the emission of the fluorophores. Andor EMCCD iXon Ultra 897 was used to record the single-molecule photon events at the frame rate of $17 \mathrm{MHz}$ and exposure time of $50 \mathrm{~ms}$, and EM gain of 300. The single-particle movie is made on a $128 \times 128$ pixel area which corresponds to the $20.48 \mu \mathrm{m}$ x $20.48 \mu \mathrm{m}$ area, and a single-pixel corresponds to the $160 \mathrm{~nm} \times 160 \mathrm{~nm}$ area. A fluorophore creates a diffraction-limited spot of $\sim 3 \times 3$ pixels, and each of these spots was analyzed as a single particle and is further used for the photon count analysis. The time trajectories were recorded and analyzed using the Andor Solis 32 bit software. The incident photons were converted to electrons and subsequent to digital counts by the EMCCD. The Andor Solis and Matlab were used to extract the total photon counts. The movies recorded under the kinetic mode of EMCCD, time/frame trajectories of the counts/intensity at a given pixel, were obtained for the provided exposure time. These time trajectories were saved for further analysis.

\section{Confocal imaging of cells labelled with three separated components}

Coverslip preparation: The glass slides and coverslips were cleaned by incubating in freshly prepared Piranha solution for $30 \mathrm{~min}$ and finally washing by MiliQ water in bath sonication then dried under nitrogen.

Cell culture, fixation and staining: HeLa cells were grown in Dulbecco's Modified Eagle Medium (DMEM) with 10\% fetal bovine serum. The cells were grown in 6-well plate on the coverslips with a density of $10^{4}$ cells per $100 \mu$ l. Each well was filled with $2 \mathrm{ml}$ of cell suspension in growth medium and the cells were allowed to grow overnight for the proper adherence and growth. The growth and the attachment of the cells to the coverslips were examined by an optical microscope. Once the cells reached proper confluency, they were fixed by incubating with $4 \%$ paraformaldehyde solution in 1x PBS buffer for 5 min. After fixation, the cells were permeabilized by 10 min incubation in $0.1 \%$ triton. The fixed and permeabilized cells were washed 4-6 times by PBS buffer to remove extra agents. These cells were incubated with the bright green, greenish brown and deep brown components at room temperature to achieve enough labelling density for confocal. The coverslips were fixed on a glass slide before imaging.

Confocal microscopy: Nikon Eclipse Ti inverted microscope was used for the confocal microscopy and images were acquired using Nikon Nis-Element software. The cell samples 
were excited by the three lasers 488, 561 and $639 \mathrm{~nm}$. Finally, the images were collected by choosing a proper filter set.

\section{Characterization of Pyrene}

FTIR: FTIR spectrum shows the characteristic vibration at $3043 \mathrm{~cm}^{-1}$ for $\mathrm{C}-\mathrm{H}$ stretching mode, $1592 \mathrm{~cm}^{-1}$ for $\mathrm{C}=\mathrm{C}$ stretching, $1181 \mathrm{~cm}^{-1}$ for $\mathrm{C}=\mathrm{C}$ bending, $832,702 \mathrm{~cm}^{-1}$ for $\mathrm{C}-\mathrm{H}$ bending.

${ }^{1}$ H NMR (500 MHz, CDCl3): $\delta 8.14(\mathrm{~d}, \mathrm{~J}=7.55 \mathrm{~Hz}, 4 \mathrm{H}), 8.04(\mathrm{~s}, 4 \mathrm{H}), 7.97$ (t, J1 = $7.6 \mathrm{~Hz}$, $\mathrm{J} 2=7.55 \mathrm{~Hz}, 2 \mathrm{H})$.

HRMS: The actual calculated $\mathrm{m} / z$ for pyrene $\left(\mathrm{C}_{10} \mathrm{H}_{16}\right)$ is 202 . The mass spectra given in figure, shows the peak with $m / z=202$.

\section{Characterization of $1,3,6$ trinitropyrene (TNP):}

After the synthesis of 1,3,6-trinitropyrene (TNP), first, we characterized the TNP using ${ }^{1} \mathrm{H}$ NMR, HRMS and FTIR.

FTIR: FTIR spectrum shows the characteristic vibration at $3080 \mathrm{~cm}^{-1}$ for $\mathrm{C}-\mathrm{H}$ stretching mode, $1580 \mathrm{~cm}^{-1}$ for $\mathrm{C}=\mathrm{C}$ vibration, $1518 \mathrm{~cm}^{-1}$ for $\mathrm{NO}_{2}$ asymmetric mode, $1348 \mathrm{~cm}^{-1}$ for $\mathrm{NO}_{2}$ symmetric mode and 845 for $\mathrm{C}-\mathrm{H}$ vibration.

${ }^{1}$ H NMR (500 MHz, DMSO-d6): $\delta 9.41$ (s, 1H), 9.23 (d, J = 10.3 Hz, 1H), 9.16 (d, J = 9.6 $\mathrm{Hz}, 1 \mathrm{H}), 9.08(\mathrm{~d}, \mathrm{~J}=9.6 \mathrm{~Hz}, 1 \mathrm{H}), 8.86(\mathrm{~d}, \mathrm{~J}=8.25 \mathrm{~Hz}, 1 \mathrm{H}), 8.59(\mathrm{~d}, \mathrm{~J}=8.9 \mathrm{~Hz}, 2 \mathrm{H})$.

HRMS: The actual calculated $m / z$ for $\mathrm{C}_{16} \mathrm{H}_{7} \mathrm{~N}_{3} \mathrm{O}_{6}$ (TNP) is 337 . The mass spectra given in figure, shows peak at $m / z=337$ and the adduct ion peak with $m / z=338\left(\mathrm{C}_{16} \mathrm{H}_{7} \mathrm{~N}_{3} \mathrm{O}_{6}+\mathrm{H}\right)$. Strong adduct ion peak with $m / z=360\left(\mathrm{C}_{16} \mathrm{H}_{7} \mathrm{~N}_{3} \mathrm{O}_{6}+\mathrm{Na}\right)$. 


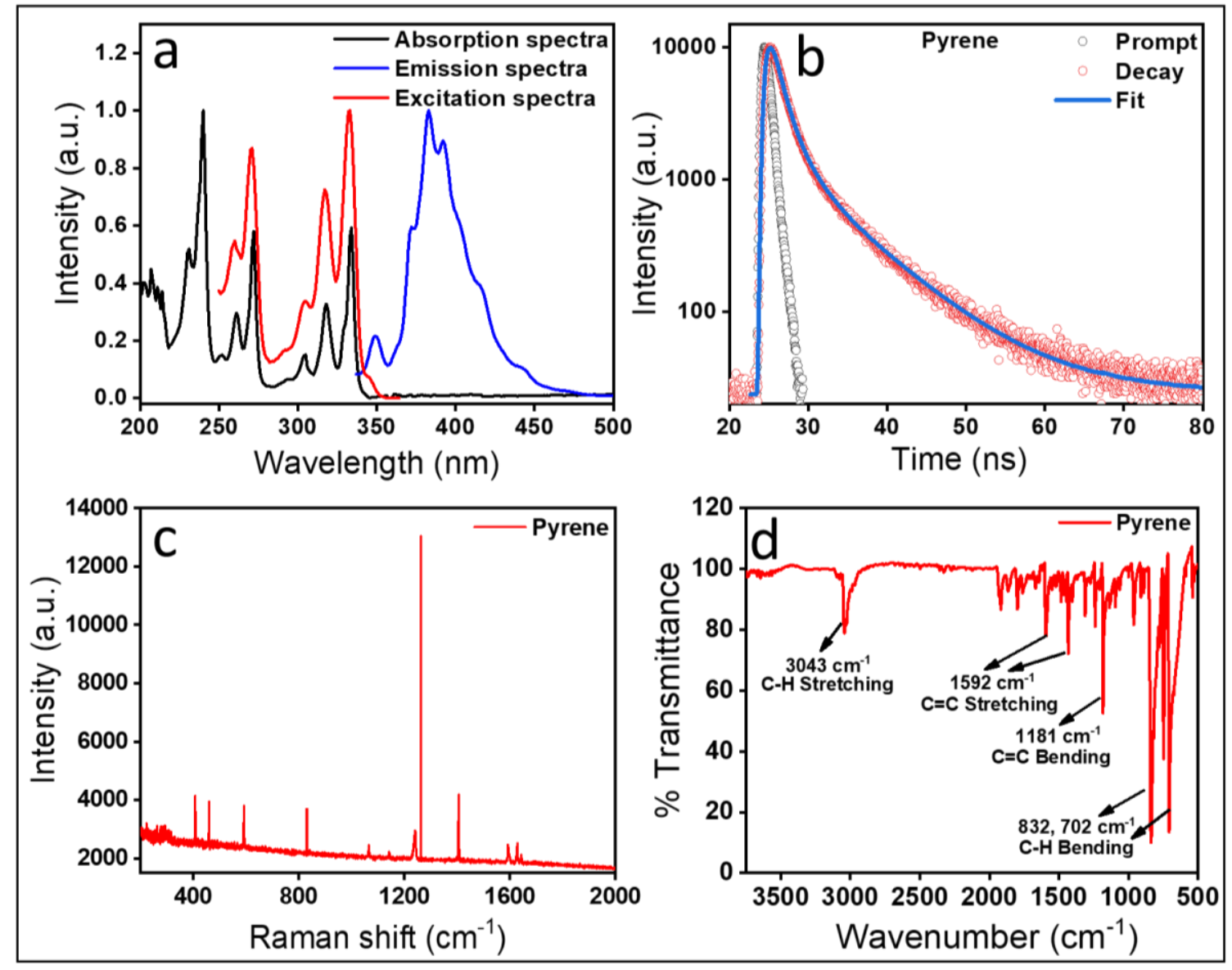

Fig. S1 (a) Absorption, emission and excitation spectra of pyrene. (b) Lifetime of pyrene with an average lifetime of $1.65 \mathrm{~ns}$. (c) Raman and (d) FTIR spectra of pyrene with the characteristics vibrational modes. 


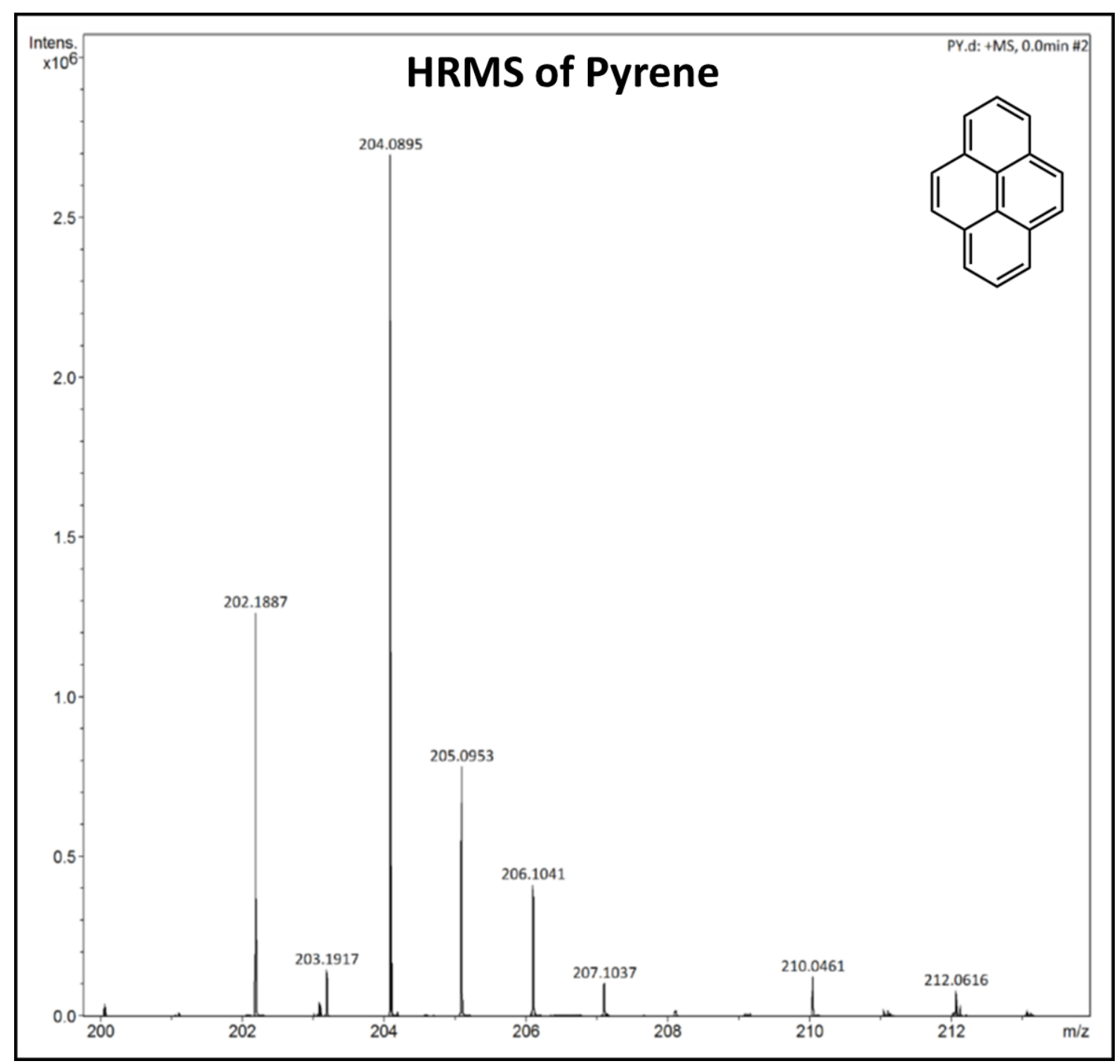

Fig. S2 HRMS spectra of pyrene. 


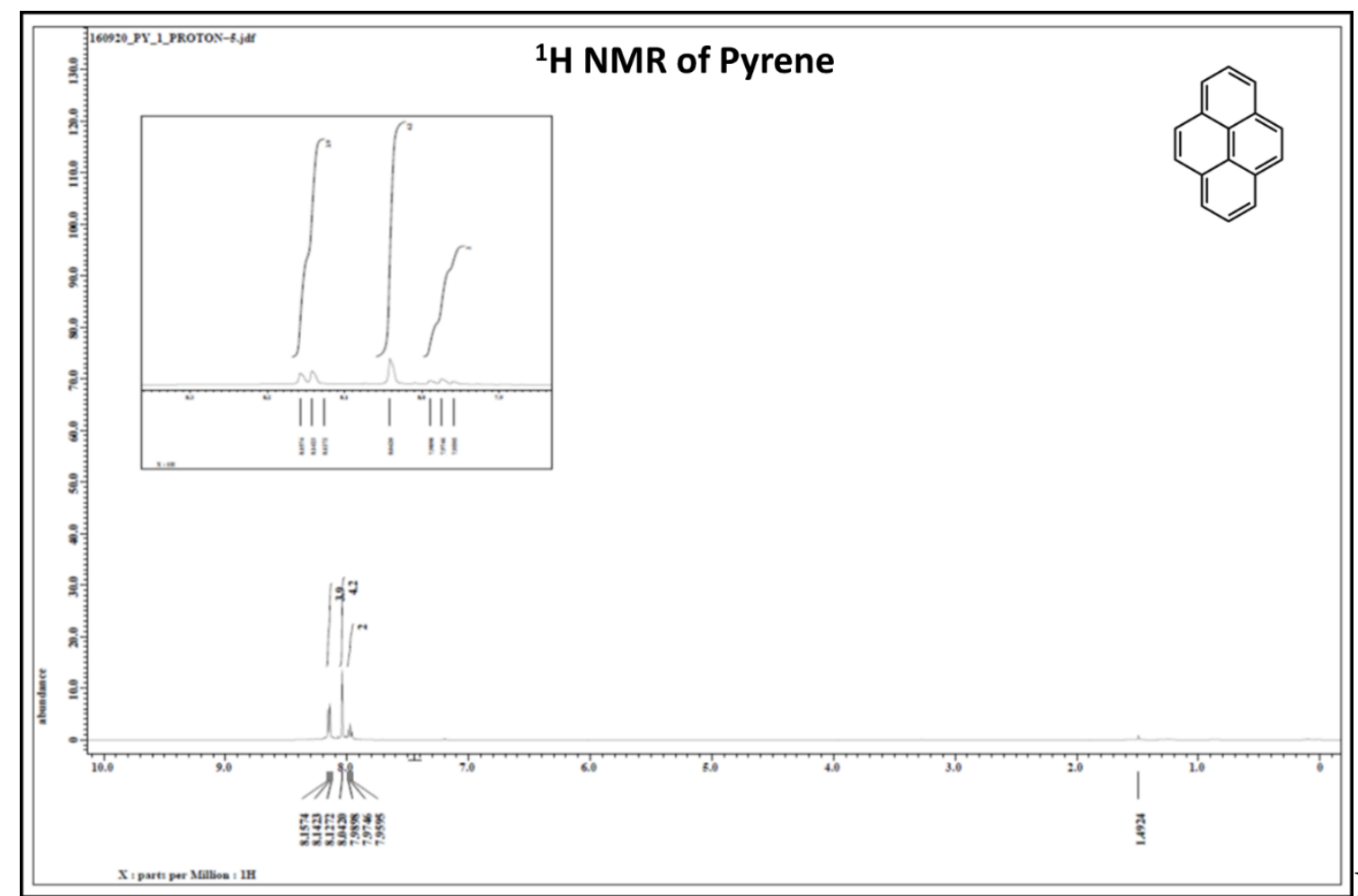

Fig. $\mathbf{S 3}{ }^{1} \mathrm{H}$ NMR spectra of pyrene.

Fig. 


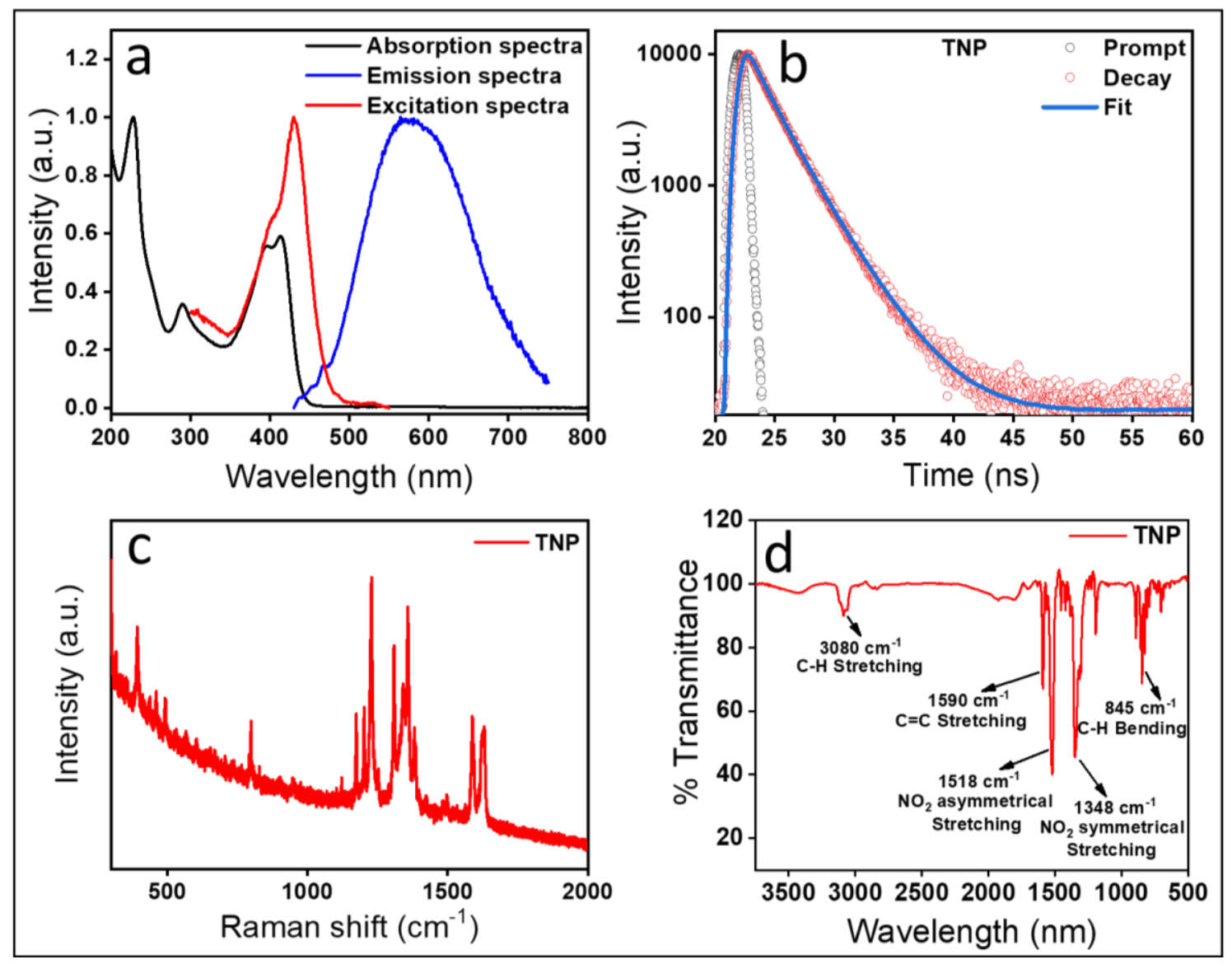

Fig. S4 (a) Absorption, emission and excitation spectra of TNP. (b) Lifetime of TNP with an average lifetime of 2.43 ns. (c) Raman spectra and (d) FTIR spectrum of TNP with the characteristics vibrational modes. 


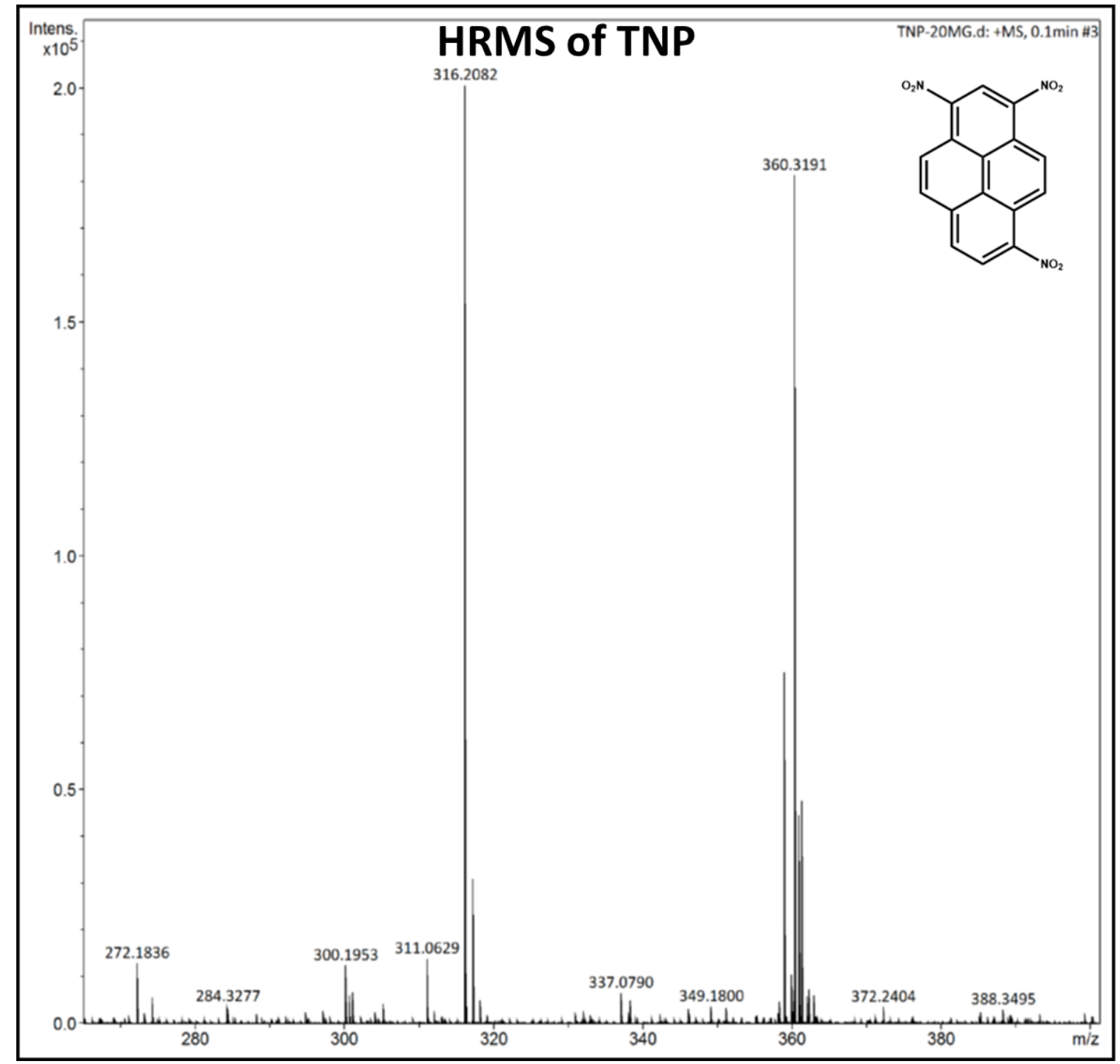

Fig. S5 HRMS spectra of TNP. 


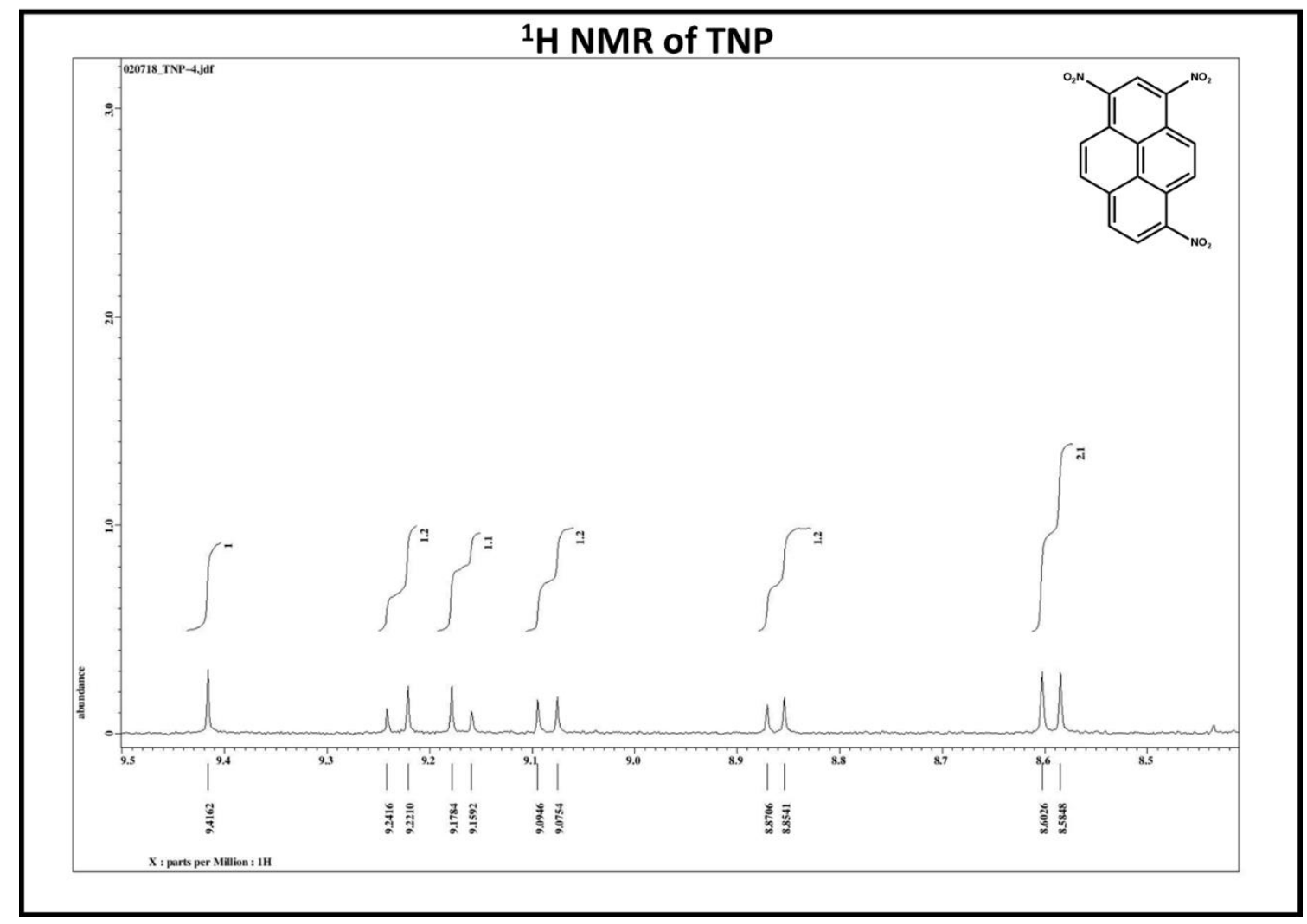

Fig. S6 ${ }^{1} \mathrm{H}$ NMR spectra of TNP. 


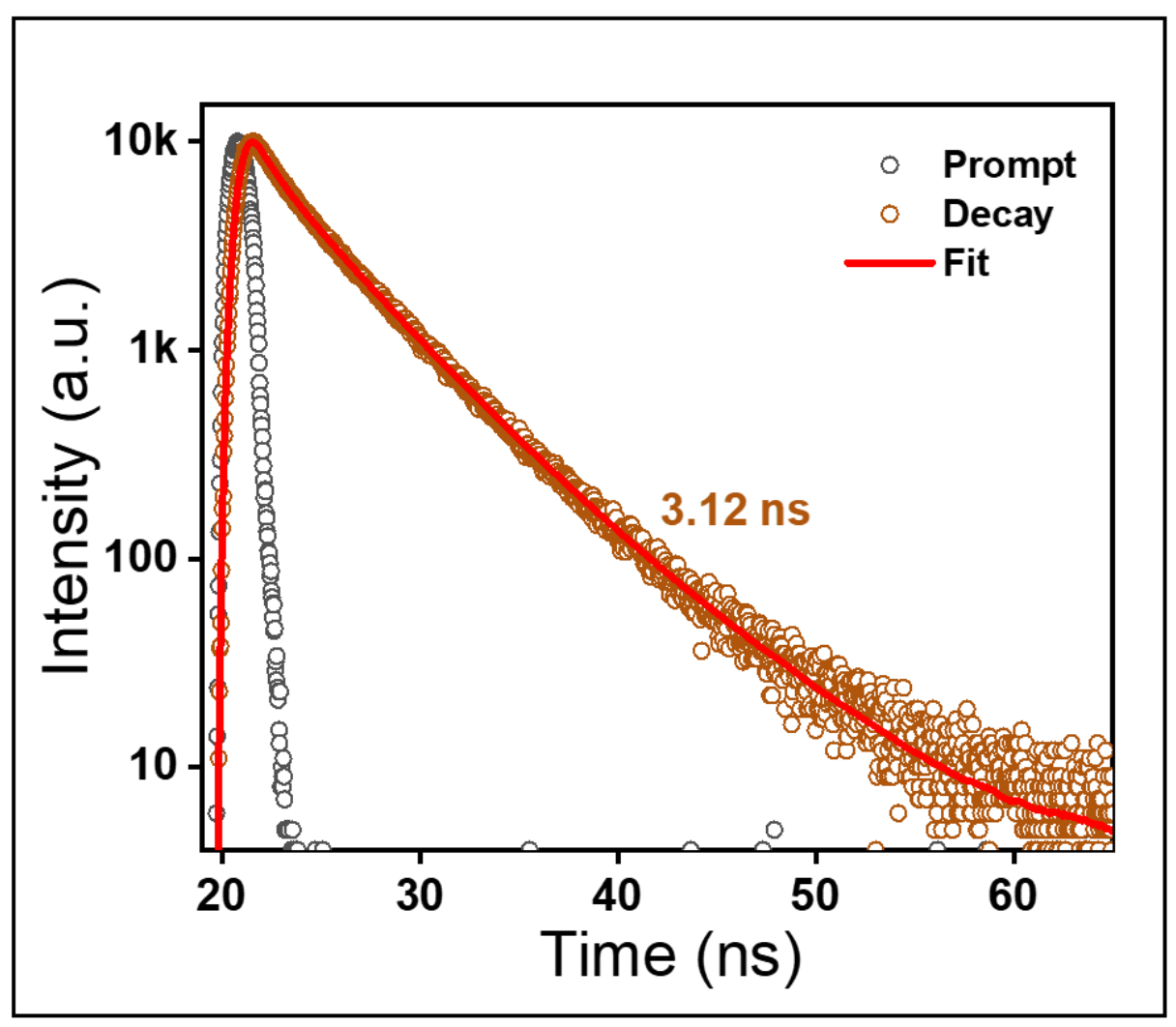

Fig. S7 Lifetime decay of crude GQDs with an average lifetime of 3.12 ns. 


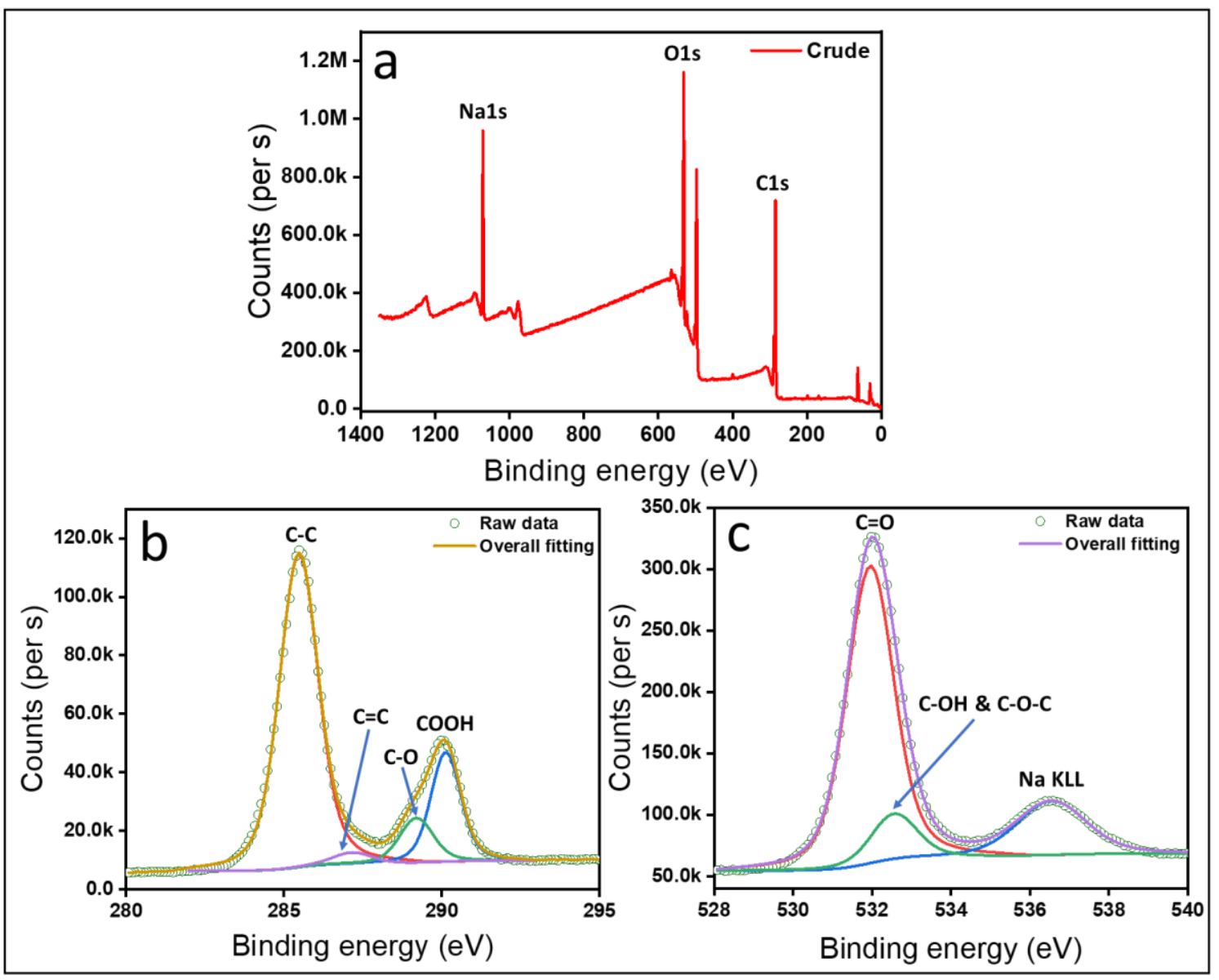

Fig. S8 (a) XPS spectra of crude GQDs. (b-c) High-resolution XPS spectra of C1s where peaks at 285.49, 287.18, 289.22 and $290 \mathrm{eV}$ representing the $\mathrm{C}-\mathrm{C}, \mathrm{C}=\mathrm{C}, \mathrm{C}-\mathrm{O}$ and $\mathrm{COOH}$ functional groups respectively. (c) High-resolution XPS spectra of O1s where peaks at 531.93 and $532.58 \mathrm{eV}$ representing the $\mathrm{C}=\mathrm{O}, \mathrm{C}-\mathrm{OH} \& \mathrm{C}-\mathrm{O}-\mathrm{C}$ functional groups. 


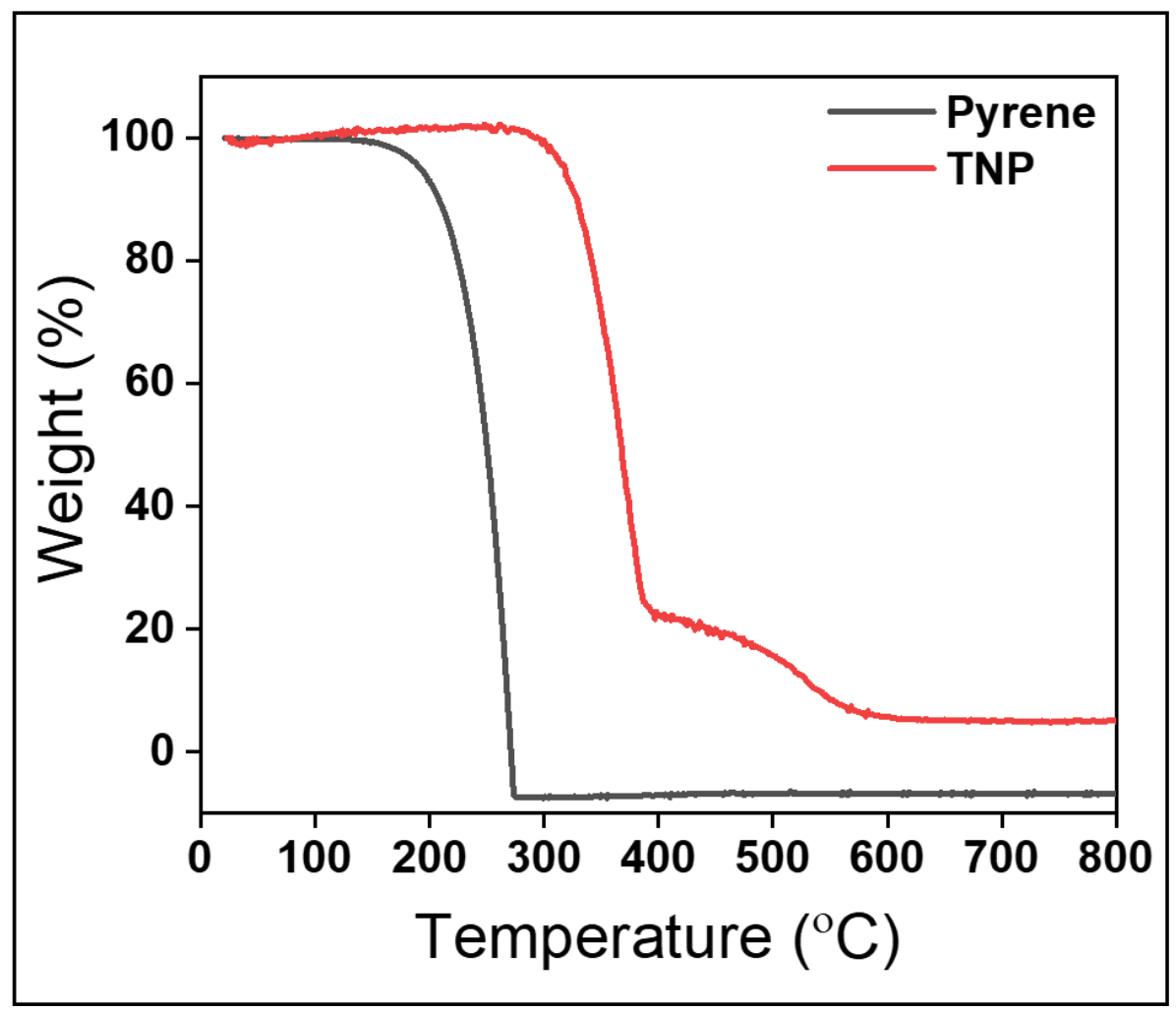

Fig. S9 Comparative TGA spectra of pyrene and TNP. A complete mass loss for pyrene was observed within $280{ }^{\circ} \mathrm{C}$, while TNP showed $80 \%$ mass loss within $400{ }^{\circ} \mathrm{C}$. 


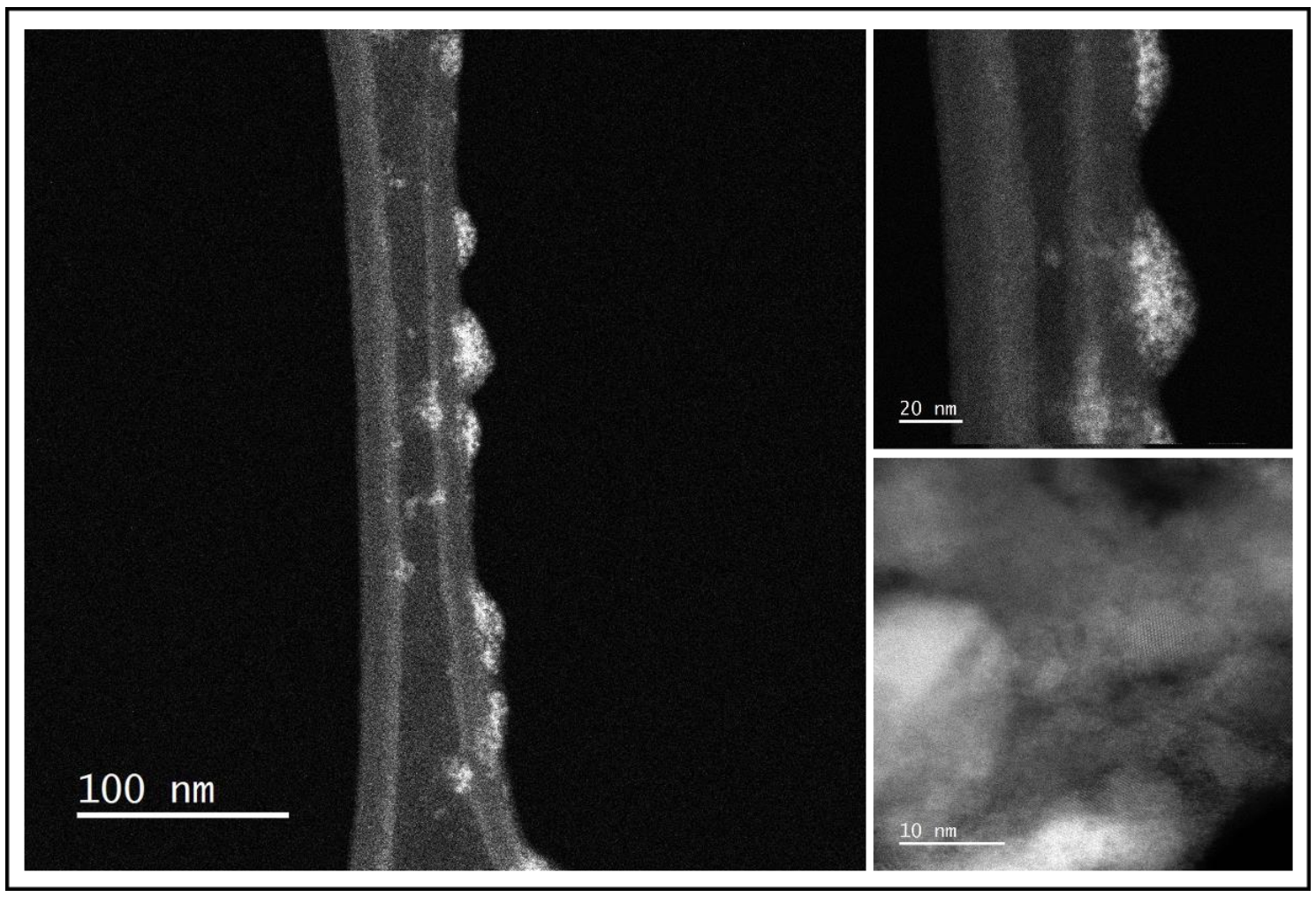

Fig. S10 STEM images of greenish brown component. Free vertically hanging organic chromophores are clearly visible on the side of the lacey carbon grid. 

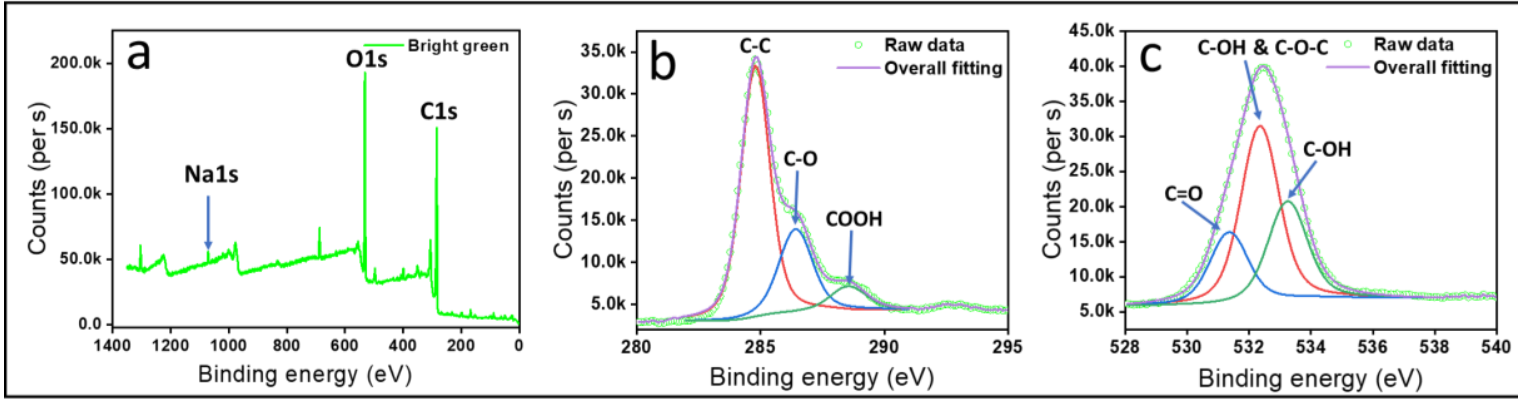

Fig. S11 (a) XPS spectra of bright green component. (b-c) High-resolution XPS spectra of $\mathrm{C} 1 \mathrm{~s}$ where peaks at $284.80,286.40,288.71$ and $289.78 \mathrm{eV}$ representing the $\mathrm{C}-\mathrm{C}, \mathrm{C}=\mathrm{C}, \mathrm{C}-\mathrm{O}$ and $\mathrm{COOH}$ functional groups respectively. (c) High-resolution XPS spectra of O1s where peaks at 531.71 and $532.67 \mathrm{eV}$ representing the $\mathrm{C}=\mathrm{O}, \mathrm{C}-\mathrm{OH} \& \mathrm{C}-\mathrm{O}-\mathrm{C}$ functional groups.
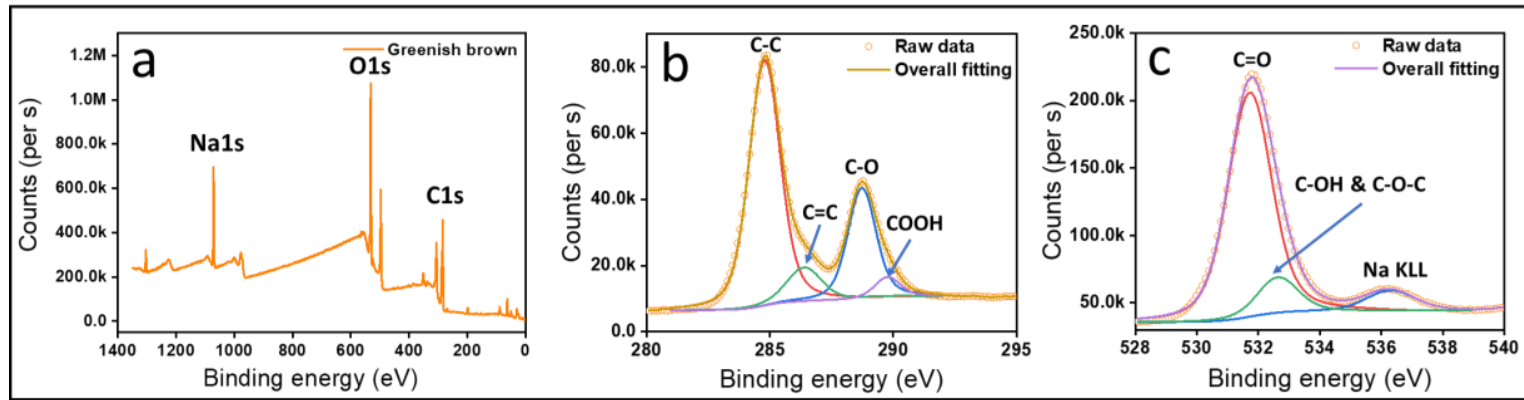

Fig. S12 (a) XPS spectra of greenish brown component. (b-c) High-resolution XPS spectra of $\mathrm{C} 1 \mathrm{~s}$ where peaks at $284.80,286.43$ and $288.60 \mathrm{eV}$ representing the $\mathrm{C}-\mathrm{C}, \mathrm{C}-\mathrm{O}$ and $\mathrm{COOH}$ functional groups respectively. (c) High-resolution XPS spectra of O1s where peaks at 531.31 and $532.35 \mathrm{eV}$ representing the $\mathrm{C}=\mathrm{O}, \mathrm{C}-\mathrm{OH} \& \mathrm{C}-\mathrm{O}-\mathrm{C}$ functional groups. 


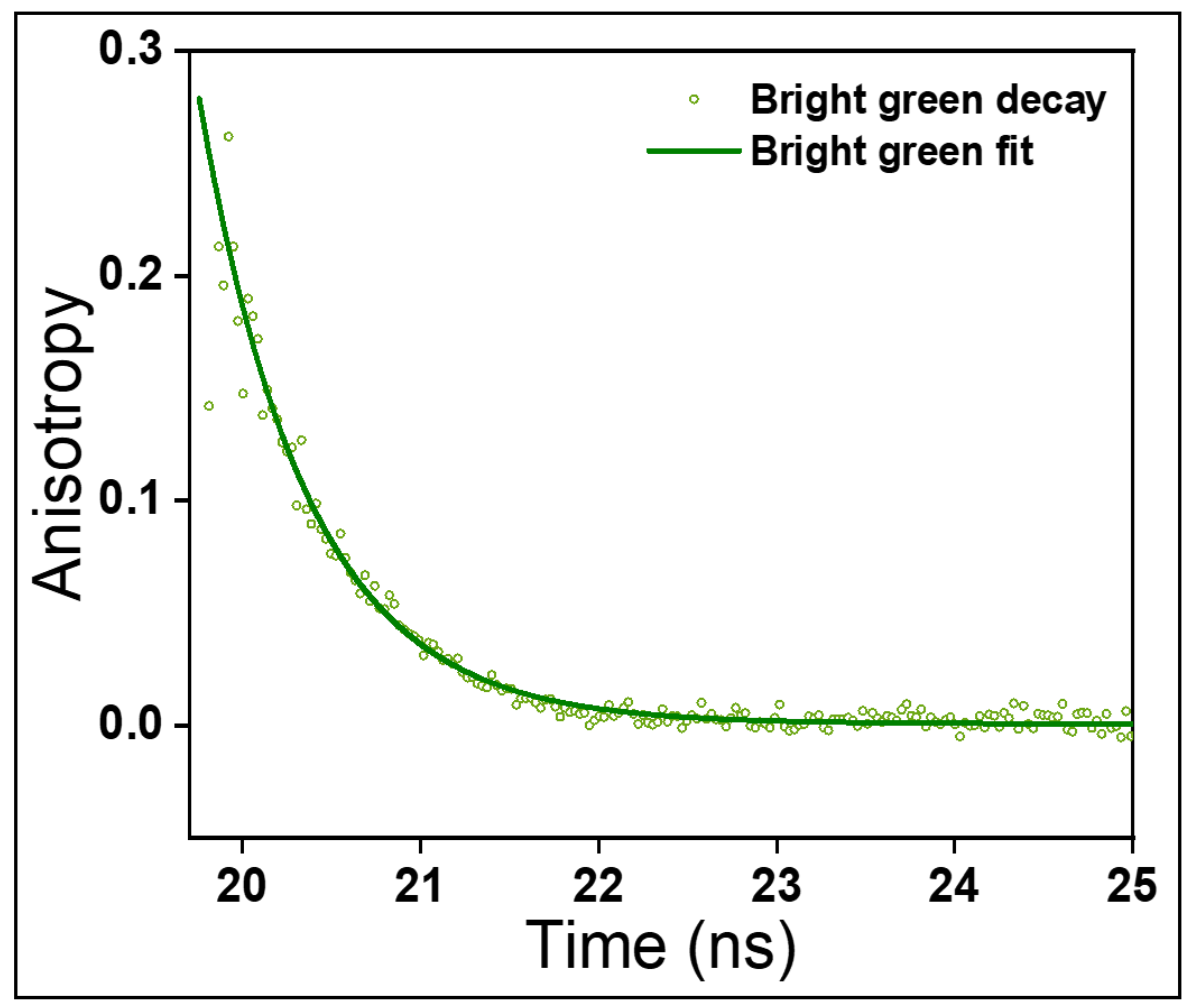

Fig. S13 Fluorescence anisotropy decay of bright green component. 


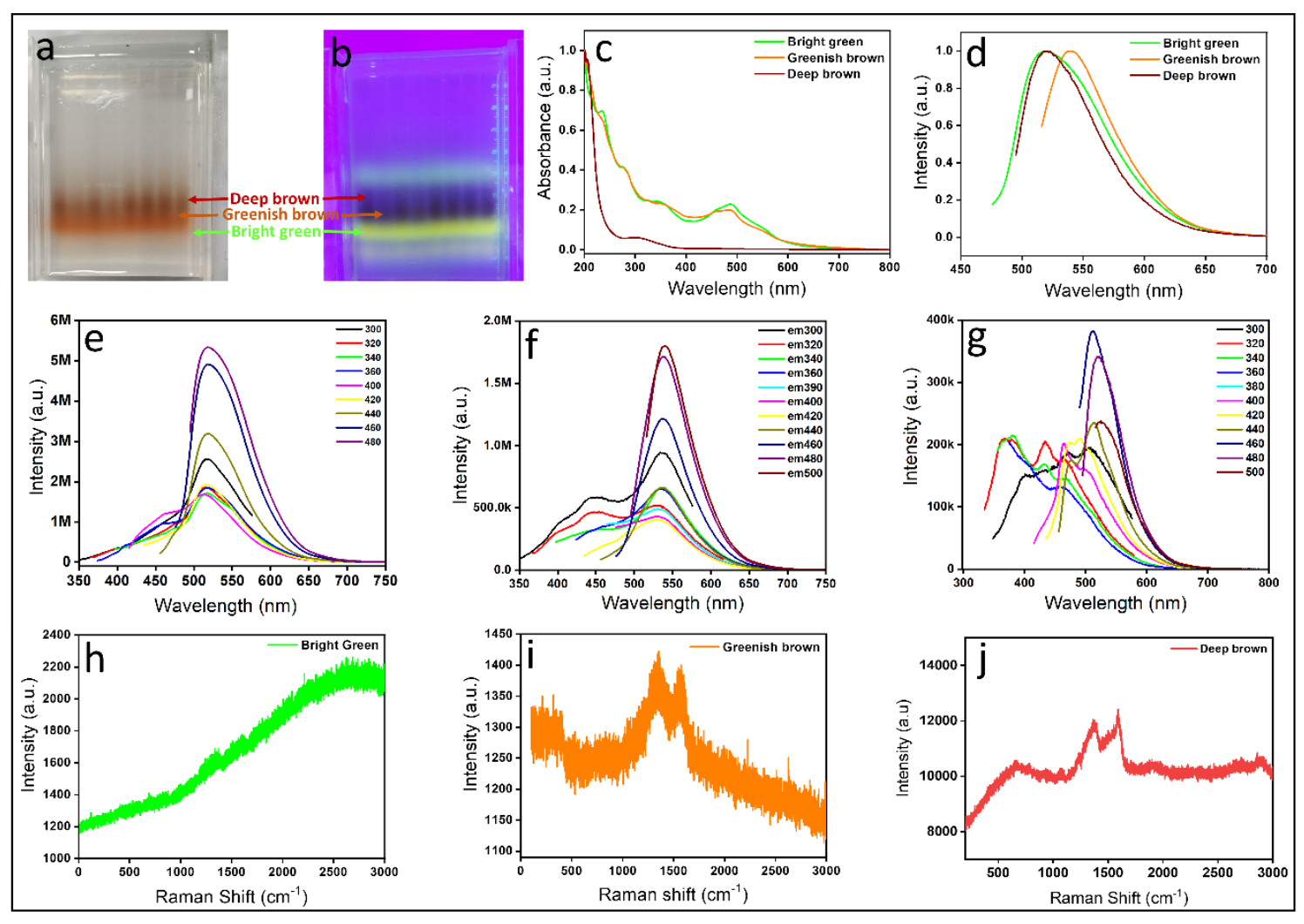

Fig. S14 Agarose gel electrophoresis separation of the synthesized GQDs. Three distinct colors both (a) in naked eye and (b) UV light illumination were observed. (c) Comparative absorption spectra of all three components. (d) Relative normalized steady-state emission spectra of all three separated components. (e-g) excitation wavelength dependent emission spectra of bright green, greenish brown and deep brown components. (h) Raman spectrum of the bright green component with fluorescence background. (i) Raman spectrum of the greenish brown component having D and $G$ bands. (j) Raman spectrum of the deep brown component having $\mathrm{D}$ and $\mathrm{G}$ bands. 

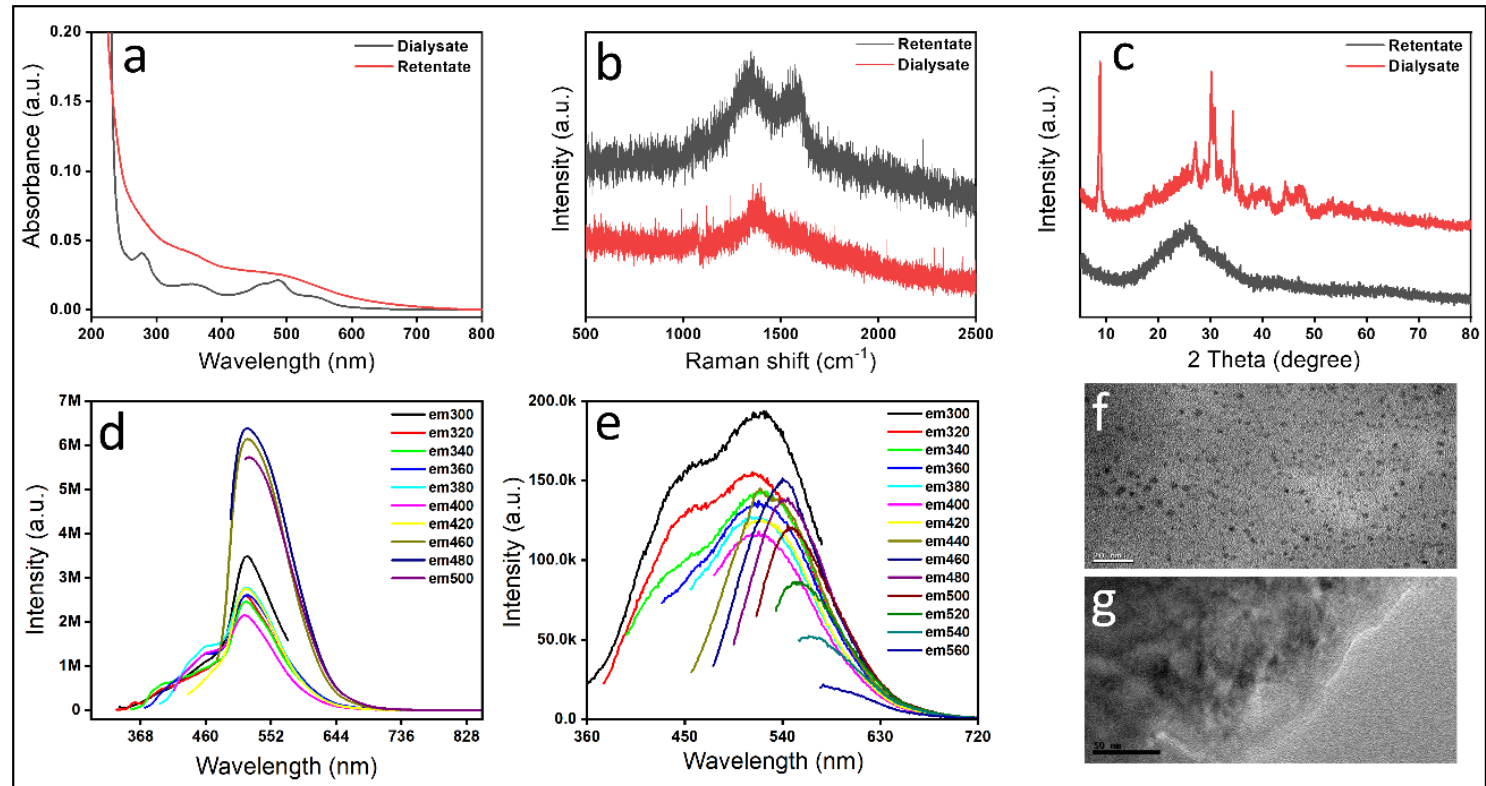

Fig. S15 (a) Absorption spectra of dialysate and retentate obtained after dialysis of crude GQDs. Dialysate showed extensive molecular state emission, while retentate showed major core and edge state with a broad molecular state emission. (b) Raman spectra of dialysate and retentate. Retentate showed D and $G$ bands but dialysate showed no D and G bands. (c) PXRD spectra of retentate and dialysate. Retentate showed broad peak, while dialysate showed structured emission with little broad peak. (d-e) Emission spectra of both dialysate and retentate excited by different wavelength. Dialysate showed excitation independent emission spectra, while retentate showed excitation dependent emission spectra. (f-g) TEM image of retentate and dialysate respectively. The retentate showed the GQDs formation with a size of $\sim 4 \mathrm{~nm}$, while the dialysate showed only aggregated structure. 


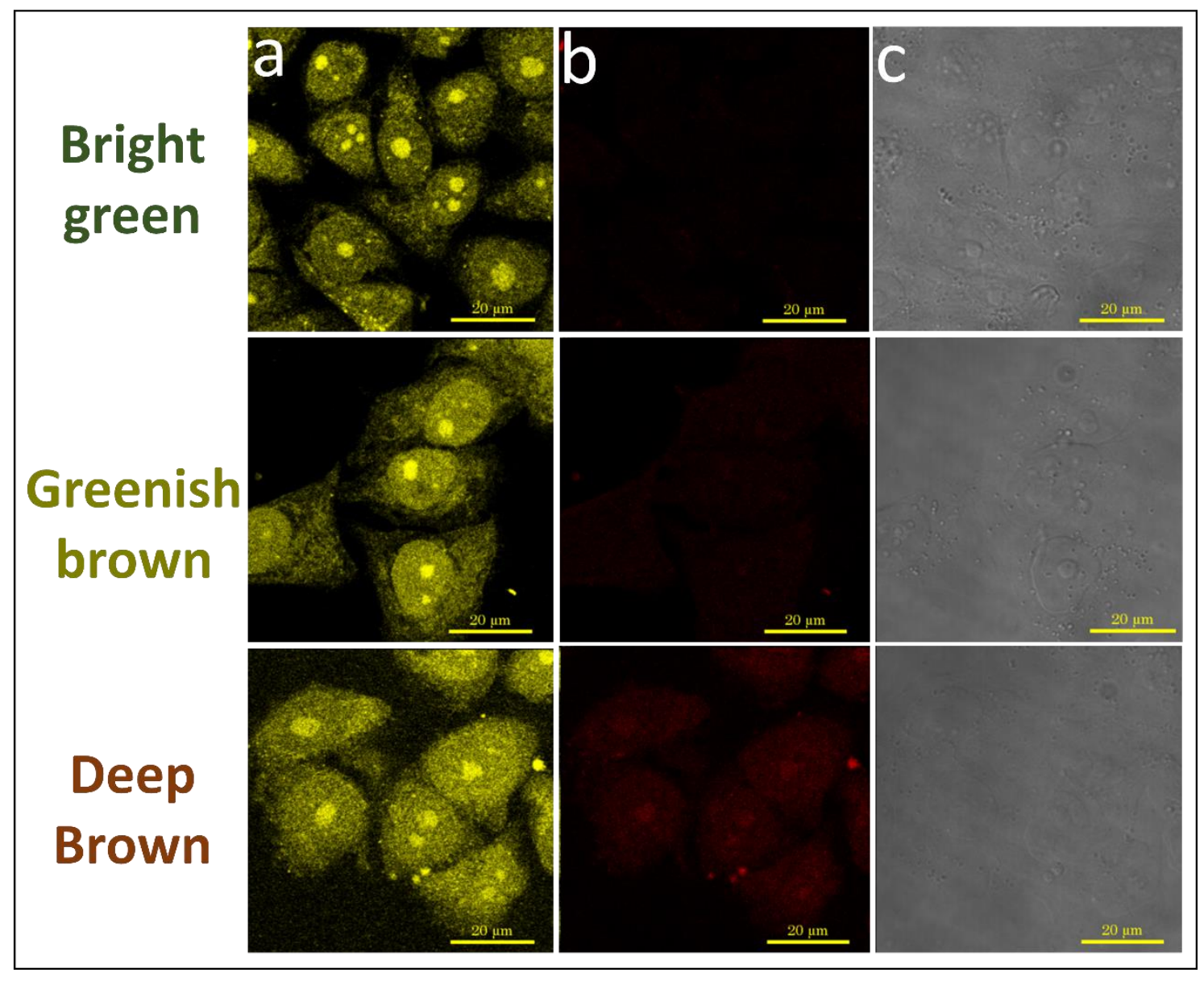

Fig. S16 (a-b) Confocal microscopy images of HeLa cells stained with bright green, greenish brown and deep brown components under different excitation laser wavelengths 488 and 639 nm. The images show that all the components have the high capability to stain the HeLa cell. Little red emission image of the cells was observed for the deep brown component. (c) TD images of the HeLa cells. 